\title{
THE MEAN-FIELD APPROXIMATION AND THE NON-LINEAR SCHRÖDINGER FUNCTIONAL FOR TRAPPED BOSE GASES
}

\author{
MATHIEU LEWIN, PHAN THÀNH NAM, AND NICOLAS ROUGERIE
}

\begin{abstract}
We study the ground state of a trapped Bose gas, starting from the full manybody Schrödinger Hamiltonian, and derive the nonlinear Schrödinger energy functional in the limit of large particle number, when the interaction potential converges slowly to a Dirac delta function. Our method is based on quantitative estimates on the discrepancy between the full many-body energy and its mean-field approximation using Hartree states. These are proved using finite dimensional localization and a quantitative version of the quantum de Finetti theorem. Our approach covers the case of attractive interactions in the regime of stability. In particular, our main new result is a derivation of the $2 \mathrm{D}$ attractive nonlinear Schrödinger ground state.
\end{abstract}

\section{Contents}

1. Introduction

2. Main results

2.1. Error bounds for the mean-field approximation

2.2. Error bounds for the Non Linear Schrödinger model

3. Error bounds for Hartree theory

3.1. Quantum de Finetti and localization

3.2. Truncated two-body Hamiltonian

3.3. Bound on the localized energy

3.4. Conclusion: proof of Theorem 2.1

4. The NLS limit

4.1. From Hartree to NLS: proof of Proposition 2.3

4.2. Convergence of the many-body energy: proof of Theorem 2.4 21

4.3. Convergence of states: proof of Theorem 2.5 21

References

\section{INTRODUCTION}

The impressive progress of cold atom physics [44, 48, during the last two decades gave a new impetus to the theory of many-bosons systems, in particular the study of Bose-Einstein condensates (BECs). In such a state of matter, many interacting particles (bosons) occupy the same quantum state and may thus be collectively modeled using a one-body nonlinear Schrödinger description.

Date: 2015. Final version to appear in the Transactions of the American Mathematical Society. (C) 2015 by the authors. 
In this framework, the energy functional of a Bose-Einstein condensate of non-relativistic particles in $1 \mathbb{R}^{d}$, interacting via a pair potential $w$, reads as (in appropriate units)

$$
\mathcal{E}_{\mathrm{H}}[u]:=\int_{\mathbb{R}^{d}}|(\nabla+i A) u|^{2}+V|u|^{2}+\frac{1}{2}|u|^{2}\left(w *|u|^{2}\right) .
$$

This so-called Hartree functional includes a trapping potential $V: \mathbb{R}^{d} \rightarrow \mathbb{R}$ confining the particles in a bounded region of space $(V(x) \rightarrow \infty$ when $|x| \rightarrow \infty)$, a feature ubiquitous in experiments with cold atoms. The vector potential $A: \mathbb{R}^{d} \rightarrow \mathbb{R}^{d}$ can model a (possibly artificial [18]) magnetic field or the Coriolis force due to the rotation of the atoms [2, 14, 23], in which case $V$ has to incorporate a contribution due to the centrifugal force. The ground state of the system is obtained by minimizing (1.1) under the mass constraint

$$
\int_{\mathbb{R}^{d}}|u|^{2}=1
$$

Samples in which Bose-Einstein condensation is achieved are typically very dilute, and it is therefore relevant to think of the case where the range of the interaction potential $w$ is much smaller than the size of the system. This situation can be modeled by using contact interactions, which amounts to formally set $w=a \delta_{0}$ proportional to the delta function at the origin. One then obtains the nonlinear Schrödinger (or Gross-Pitaevskii) functional

$$
\mathcal{E}_{\mathrm{nls}}[u]:=\int_{\mathbb{R}^{d}}|(\nabla+i A) u|^{2}+V|u|^{2}+\frac{a}{2}|u|^{4} .
$$

Here $a$ measures the strength of interparticle interactions. An appealing aspect of cold atoms experiments is the possibility to tune the value and even the sign of $a$, going from repulsive $(a>0)$ to attractive interactions $(a<0) 2$. In the latter case the system may collapse, i.e. there might not exist a ground state for (1.3). It is in fact the case if $d=2$ and $a \leq-a^{*}$ for some critical value $a^{*}>0$, and also if $d \geq 3$ and $a<0$.

An important question is that of the relation between the macroscopic, nonlinear description using functionals such as (1.1) and (1.3), and the underlying, linear, physics based on the many-body Schrödinger equation. In the latter model the system is described via a many-body Hamiltonian of the form

$$
H_{N}=\sum_{j=1}^{N}\left(-\left(\nabla_{x_{j}}+i A\left(x_{j}\right)\right)^{2}+V\left(x_{j}\right)\right)+\frac{1}{N-1} \sum_{1 \leq i<j \leq N} N^{d \beta} w\left(N^{\beta}\left(x_{i}-x_{j}\right)\right)
$$

acting on $\mathfrak{H}^{N}:=\bigotimes_{s}^{N} \mathfrak{H}$, the symmetric tensor product of $N$ copies of the one-body Hilbert space $\mathfrak{H}=L^{2}\left(\mathbb{R}^{d}\right)$ describing quantum particles living in $d$ dimensions. The symmetry restriction is necessary for bosonic particles that correspond to square integrable complex wave functions $\Psi \in L^{2}\left(\mathbb{R}^{d N}\right) \simeq \bigotimes^{N} L^{2}\left(\mathbb{R}^{d}\right)$ of $N$ variables satisfying

$$
\Psi\left(x_{1}, \ldots, x_{N}\right)=\Psi\left(x_{\sigma(1)}, \ldots, x_{\sigma(N)}\right)
$$

for any permutation $\sigma$. The ground state energy associated with (1.4) is simply the smallest eigenvalue of the operator, and a ground state is any associated eigenfunction. Roughly

\footnotetext{
${ }^{1}$ We are interested in $d=1,2,3$ but our method also applies to $d \geq 4$.

${ }^{2}$ Respectively from defocusing to focusing interactions in the quantum optics vocabulary.
} 
speaking, one should expect that when $N \rightarrow \infty$, a ground state of (1.4) factorizes:

$$
\Psi\left(x_{1}, \ldots, x_{N}\right) \approx \prod_{j=1}^{N} u\left(x_{j}\right),
$$

with $u \in L^{2}\left(\mathbb{R}^{d}\right)$ a ground state of the nonlinear one-body theory. Of course (1.6) must be taken with care: determining in which precise sense it holds is a subtle task that has motivated much research.

In (1.4) we have scaled the interaction term in such a way that we may expect a welldefined theory in the limit $N \rightarrow \infty$. The prefactor $(N-1)^{-1}$ ensures that the interaction energy stays of order $N$, and the fixed parameter $0 \leq \beta \leq 1$ allows to model different interacting scenarii by changing the $N$-dependence of the potential range. For illustration, let us recall how the limit problem depends on $\beta$ in three space dimensions:

- If $\beta=0$ we are in the mean-field (MF) regime. The range of the interaction potential is fixed but its intensity goes to zero proportionally to $N^{-1}$. In this case the limit problem is (1.1). The relation between (1.1) and (1.4) has been clarified under special assumptions on $w$ in $[7,41,53,26,54$ ] and in full generality in [32, taking inspiration from the earlier works $21,49,59$.

- If $0<\beta<1$, the scaled interaction potential converges in the sense of measures to a delta function

$$
w_{N}:=N^{d \beta} w\left(N^{\beta} \cdot\right) \rightarrow\left(\int_{\mathbb{R}^{d}} w\right) \delta_{0}
$$

and the limit problem becomes (1.3) with $a=\int_{\mathbb{R}^{d}} w$. We will refer to this case as the nonlinear Schrödinger (NLS) limit. It has not been considered much in the literature. However, when $w \geq 0$, the techniques invented to tackle the harder case $\beta=1$ a fortiori apply in this case.

- If $\beta=1$, the interaction potential of course still converges to a delta function but, in dimension $d=3$, the limit functional is now (1.3) with $a=8 \pi \times$ (scattering length of $w$ ). This is due to the fact that the ground state of (1.4) includes a non trivial correction to the ansatz (1.6), in the form of a short scale correlation structure. The relationship between (1.4) and (1.3) in this so-called Gross-Pitaevskii (GP) limit has been established by Lieb, Seiringer and Yngvason in a seminal series of papers (we refer for example to [42, 38, 40, 35], or [37] for a review, see also [8]), assuming the interactions to be purely repulsive, $w \geq 0$.

The corresponding evolution problems have also attracted a lot of attention recently. Among many references, one may consult [6, 4, 25, 50, 47] for the MF limit, [1, 19, 46] for the NLS limit and [20, 45] for the GP limit. For the derivation of the 1D focusing NLS equation, we refer to [10].

The purpose of this paper is to present a new method allowing to rigorously establish that the minimization of (1.3) correctly describes the ground state of (1.4) in the limit $N \rightarrow \infty$. We are limited to rather small values of $\beta$, i.e. to potentials converging slowly (with polynomial rate) to delta interactions, so we always obtain (1.3) with

$$
a=\int_{\mathbb{R}^{d}} w
$$


Our method consists in establishing quantitative bounds on the difference between the $N$-body energy per particle and an $N$-dependent Hartree energy obtained by taking

$$
w_{N}=N^{d \beta} w\left(N^{\beta} .\right)
$$

as an interaction potential in (1.1). The NLS functional is obtained in a second step by passing to the limit in the Hartree functional. We thus treat separately the issues of the mean-field approximation (the validity of the ansatz (1.6)) and that of the short range of the interactions (the nature of the one-body state $u$ in the ansatz (1.6)).

To deal with the first (main) issue we elaborate on the method we presented in [32], which is based on the quantum de Finetti theorem [57, 29]. However, in [32] we relied heavily on compactness arguments and thus did not obtain any error bound, which is required to deal with a $N$-dependent interaction potential as we do here. Our strategy in this paper is to use the localization method in Fock space described in [30] to reduce the problem to a finite dimensional setting in which we may employ a quantitative version of the quantum de Finetti theorem due to Christandl, König, Mitchison and Renner [12].

The intuition behind this procedure is as follows. Under the assumption that the system is trapped, namely $V(x) \rightarrow \infty$ when $|x| \rightarrow \infty$, the one-body operator

$$
H_{1}:=-(\nabla+i A(x))^{2}+V(x)
$$

of the Hamiltonian (1.4) has a discrete spectrum made of a diverging sequence of eigenvalues. In the ground state of the $N$-body Hamiltonian, the number of particles living on one-body states with high energy will clearly be small. The idea is thus to restrict our attention to the subsystem consisting of all the particles which have a one-particle energy below a given cut-off $L$, that is allowed to tend to infinity slowly.

The part of the many-body ground state living in the low energy space will be dealt with using a quantitative version of the finite dimensional quantum de Finetti theorem 22, 12, 11, 28, 31]. This result roughly says that the reduced density matrices of any $N$-body state can be approximated by that of a convex combination of product states $\left|u^{\otimes N}\right\rangle\left\langle u^{\otimes N}\right|$. The energy being a linear functional of the 2-body density matrix, it is then easy to obtain the Hartree energy as a lower bound. The approximation error due to this procedure is proportional to the dimension of the low-lying energy space and inversely proportional to the number of particles. A crucial step therefore consists in optimizing over the energy cut-off $L$ (which governs the dimension of the low energy subspace) to minimize the error due to the use of the de Finetti theorem.

A related approach was used by Lieb and Seiringer in 35] who dealt with the GrossPitaevskii limit of the same model. After having replaced the interaction by a smeared one involving the scattering length (a step which is not considered here), they also localized the particles to the lower energy states. Then they used coherent states in Fock space for the particles with the lower energies, in order to recover the mean-field functional. An advantage of our approach is that it works "locally in Fock space", that is, in any $n$-particle subspace independently of the others and thus avoids some difficulties related to the control of high values of the particle number.

Our method seems less sensitive to the type of interaction potential considered than previous approaches. In particular we do not need $w \geq 0$ to establish quantitative estimates on the difference between the $N$-body energy and the $N$-dependent Hartree energy. However, we need a sufficiently stable system to obtain the NLS energy when passing to the limit 
$N \rightarrow \infty$. In fact, the $N$-body system must be stable of the second kind, namely the lowest energy per particle must be uniformly bounded. In the following, we shall only use stability conditions for the $N$-dependent Hartree functional. Since the Hartree energy is always an upper bound to the $N$-body energy, the Hartree stability is obviously necessary for the $N$-body stability. That the Hartree stability is sufficient for the $N$-body stability is not obvious, and it comes from our quantitative estimates on the energy difference between the $N$-body and Hartree models. To be precise, for $\beta$ small enough (depending on $d$ and the trapping potential $V$ ), we obtain the NLS energy (and NLS ground state) from the $N$-body model when $N \rightarrow \infty$ in the following cases

(1) $d=3$ and the interaction potential is stable in the sense that

$$
\iint_{\mathbb{R}^{3} \times \mathbb{R}^{3}}|u(x)|^{2}|u(y)|^{2} w(x-y) d x d y \geq 0, \quad \forall u \in L^{2}\left(\mathbb{R}^{d}\right) .
$$

Assumption (1.9) is necessary in dimensions $d \geq 3$. In fact, (1.9) is necessary (and sufficient if $w$ is regular enough) for the stability of the second kind of classical particles interacting via the potential $w$, see Subsection 2.2.1 below.

(2) $d=2$ and the potential is stable in the sense that

$$
\inf _{u \in H^{1}\left(\mathbb{R}^{2}\right)}\left(\frac{\iint_{\mathbb{R}^{2} \times \mathbb{R}^{2}}|u(x)|^{2}|u(y)|^{2} w(x-y) d x d y}{2\|u\|_{L^{2}\left(\mathbb{R}^{2}\right)}^{2}\|\nabla u\|_{L^{2}\left(\mathbb{R}^{2}\right)}^{2}}\right)>-1 .
$$

Except for the case of equality, this assumption is again also necessary.

(3) $d=1$ under no specific assumption on the interaction potential.

See Section 2.2.1 for a detailed discussion on these assumptions. Of these three cases, the second is the one that presents the main novelty of the paper: in $2 \mathrm{D}$, we present the first derivation of the attractive NLS ground state in the regime of stability. In 3D on the other hand our conditions on $\beta$ are much more stringent than those in [38, 35] where the GP limit is covered in the case $w \geq 0$. The result is thus not new but our method of proof may still be of interest, in particular because it provides error bounds, and treats on the same footing the case with and without vector potential $A$. In 1D, because of the Sobolev embedding, one may directly use contact interactions at the level of the many-body Hamiltonian (1.4) as in [34, 54, so the procedure of scaling a regular interaction potential is less relevant. However, we provide a derivation of the attractive $1 \mathrm{D}$ ground state with what seems to be an unprecedented precision on the error bound.

Our main theorems are stated in the next section, and their proofs occupy the rest of the paper.

Acknowledgement: The authors acknowledge financial support from the European Research Council (FP7/2007-2013 Grant Agreement MNIQS 258023) and the ANR (Mathostaq project, ANR-13-JS01-0005-01). PTN and NR have benefited from the hospitality of the Institute for Mathematical Science of the National University of Singapore. 


\section{MAIN RESUltS}

We do not aim at optimal assumptions on the potentials $V$ and $w$. We will assume that $w$ is integrable, symmetric and uniformly bounded:

$$
w \in L^{1}\left(\mathbb{R}^{d}, \mathbb{R}\right), \quad w(x)=w(-x) \quad \text { and } \quad|w(x)| \leq C .
$$

We shall denote by

$$
w_{N}(x):=N^{d \beta} w\left(N^{\beta} x\right)
$$

the scaled interaction potential, and use the same notation $w_{N}$ for the operator acting on the two-particle space $\mathfrak{H}^{2} \simeq L^{2}\left(\mathbb{R}^{2 d}\right)$ as the multiplication by $\left(x_{1}, x_{2}\right) \mapsto w_{N}\left(x_{1}-x_{2}\right)$.

The fact that we consider a trapped system, as appropriate for experiments with cold atomic gases, is materialized by the following assumption on the one-body potential:

$$
V \in L_{\text {loc }}^{1}\left(\mathbb{R}^{d}, \mathbb{R}\right), \quad V(x) \geq c|x|^{s}-C
$$

for some exponent $s>0$ and some constants $c>0, C \geq 0$. Our estimates will essentially involve the exponent $s$.

As for the vector potential $A$, that can model a magnetic field or Coriolis forces acting on rotating particles, it is sufficient to assume

$$
A \in L_{\mathrm{loc}}^{2}\left(\mathbb{R}^{d}, \mathbb{R}^{d}\right) .
$$

A particularly relevant example is given by $A(x)=\Omega\left(-x_{2}, x_{1}, 0\right)$ for $d=3$ and $A(x)=$ $\Omega\left(-x_{2}, x_{1}\right)$ for $d=2$, corresponding to Coriolis forces due to a rotation at speed $\Omega$ around the $x_{3}$-axis, or a constant magnetic field of strength $\Omega$ pointing in this direction.

2.1. Error bounds for the mean-field approximation. Our first main task is to provide quantitative bounds on the discrepancy between the ground state energy per particle corresponding to (1.4)

and the nonlinear energy

$$
E(N):=\inf \sigma_{\mathfrak{H}^{N}} H_{N}
$$

$$
e_{\mathrm{H}}:=\inf _{\|u\|_{L^{2}\left(\mathbb{R}^{d}\right)}=1} \mathcal{E}_{\mathrm{H}}[u]
$$

given by the minimization of the Hartree functional

$$
\begin{aligned}
\mathcal{E}_{\mathrm{H}}[u]:=\int_{\mathbb{R}^{d}}\left(|(\nabla+i A(x)) u(x)|^{2}+V(x)|u(x)|^{2}\right) d x & \\
& +\frac{1}{2} \iint_{\mathbb{R}^{d} \times \mathbb{R}^{d}}|u(x)|^{2} w_{N}(x-y)|u(y)|^{2} d x d y .
\end{aligned}
$$

Note that when $\beta>0$ both $\mathcal{E}_{\mathrm{H}}$ and $e_{\mathrm{H}}$ depend on $N$ and that $\left|e_{\mathrm{H}}\right| \leq C N^{d \beta}$.

It will be convenient to introduce a slightly modified Hartree energy with the interaction $w_{N}$ replaced by $w_{N}-\varepsilon\left|w_{N}\right|$ for some $\varepsilon>0$ (that will later on be taken small enough):

$$
\mathcal{E}_{\mathrm{H}}^{\varepsilon}[u]:=\mathcal{E}_{\mathrm{H}}[u]-\frac{\varepsilon}{2} \iint_{\mathbb{R}^{d} \times \mathbb{R}^{d}}|u(x)|^{2}\left|w_{N}(x-y)\right||u(y)|^{2} d x d y .
$$

We denote by

$$
e_{\mathrm{H}}^{\varepsilon}:=\inf _{\|u\|_{L^{2}\left(\mathbb{R}^{d}\right)}=1} \mathcal{E}_{\mathrm{H}}^{\varepsilon}[u]
$$

the corresponding ground state energy, which satisfies $\left|e_{\mathrm{H}}^{\varepsilon}\right| \leq C(1+\varepsilon) N^{d \beta}$. 
When $\beta>0$ we also consider the NLS energy functional

$$
\mathcal{E}_{\mathrm{nls}}[u]=\int_{\mathbb{R}^{d}}\left(|(\nabla+i A(x)) u(x)|^{2}+V(x)|u(x)|^{2}\right) d x+\frac{a}{2} \int_{\mathbb{R}^{d}}|u(x)|^{4} d x
$$

with ground state energy

$$
e_{\mathrm{nls}}=\inf _{\|u\|_{L^{2}\left(\mathbb{R}^{d}\right)}=1} \mathcal{E}_{\mathrm{nls}}[u]
$$

that arises as the limit $N \rightarrow \infty$ of (2.6). As announced, $a$ will always be defined as

$$
a:=\int_{\mathbb{R}^{d}} w
$$

The ground state energy $e_{\text {nls }}$ is finite in dimensions $d \geq 2$ only under appropriate assumptions on $a$ (e.g. $a \geq 0)$.

For $\beta=0$ it is well-known (see [32] and references therein) that

$$
\lim _{N \rightarrow \infty} \frac{E(N)}{N}=e_{\mathrm{H}}
$$

For the purpose of this paper we need to provide explicit estimates in the case of a confined system and for $\beta \geq 0$, which is the content of the following

Theorem 2.1 (Error bounds for the mean-field approximation). We assume that (2.1), (2.2) and (2.3) hold true.

- If $d=1$ and $\beta>0$, then we have

$$
e_{\mathrm{H}} \geq \frac{E(N)}{N} \geq e_{\mathrm{H}}-C N^{-\frac{1}{4+2 / s}} .
$$

- If $d=1$ and $\beta=0$, or if $d \geq 2$ and

$$
0 \leq \beta<\frac{1}{d(1+d / s+d / 2)},
$$

then we have

$$
e_{\mathrm{H}} \geq \frac{E(N)}{N} \geq e_{\mathrm{H}}^{\varepsilon}-C \frac{\varepsilon^{-1-d / 2-d / s}}{N^{1-d \beta(1+d / 2+d / s)}},
$$

for all $0<\varepsilon \leq 1$.

Without more information on the potential $w$ and when $\beta>0$, it is not obvious that the problem $e_{\mathrm{H}}^{\varepsilon}$ is actually close to $e_{\mathrm{H}}$ and we will only be able to go further under some additional stability assumptions on $w$. We can however make the following

Remark 2.2 (Estimates in the mean-field limit).

Using the simple estimate

$$
e_{\mathrm{H}}^{\varepsilon} \geq e_{\mathrm{H}}-C N^{d \beta} \varepsilon
$$

and optimizing with respect to $\varepsilon$, we can immediately deduce from (2.15) a bound which is valid for a smaller range of $\beta$ but does not involve $e_{\mathrm{H}}^{\varepsilon}$ anymore. Namely, if

$$
0 \leq \beta<\frac{1}{d(2+d / s+d / 2)},
$$

then we have

$$
e_{\mathrm{H}} \geq \frac{E(N)}{N} \geq e_{\mathrm{H}}-C N^{d \beta-\frac{1}{2+d / s+d / 2}} .
$$


If $\beta=0$ then $e_{\mathrm{H}}$ is independent of $N$ and we have thus the uniform bound

$$
e_{\mathrm{H}} \geq \frac{E(N)}{N} \geq e_{\mathrm{H}}-C N^{-\frac{1}{2+d / 2+d / s}}
$$

which gives a bound on the rate of convergence in (2.12).

With more information on $w$, it is clear that (2.18) is not optimal. Indeed, it has been proved in [33, 53, 26] that when the Hartree minimization problem $e_{\mathrm{H}}$ has a unique nondegenerate minimizer, then the next order is given by Bogoliubov's theory and it is of order $1 / N$. This is for instance the case $\mathrm{i} \hat{3} \widehat{w}>0$ and the vector potential $A$ is small enough. Without more assumptions on $w$, we are not aware of any existing quantitative error bound. For instance, in a gas rotating sufficiently fast for vortices to be nucleated (see [15, 16, 17, 52. and references therein), uniqueness is known to fail and our bound (2.18) seems to be the best so far.

For illustration, we compute the rate of convergence we obtain in a few physically interesting situations (see Table 1), still in the Hartree case $\beta=0$. We consider space dimensions $d=1,2,3$, and compare the harmonic oscillator case $s=2$ with the case of particles in a box where we set formally $s=\infty$. In fact our method also applies to the case of particles confined to a bounded domain and we obtain the rates for $s=\infty$ in this case.

\begin{tabular}{|c|c|c|c|}
\hline & $d=3$ & $d=2$ & $d=1$ \\
\hline$s=2$ & $N^{-1 / 5}$ & $N^{-1 / 4}$ & $N^{-1 / 3}$ \\
\hline$s=\infty$ & $N^{-2 / 7}$ & $N^{-1 / 3}$ & $N^{-2 / 5}$ \\
\hline
\end{tabular}

TABle 1. Rates of convergence to Hartree's energy $(\beta=0)$.

2.2. Error bounds for the Non Linear Schrödinger model. If $\beta>0$, then $e_{\mathrm{H}}$ and $e_{\mathrm{H}}^{\varepsilon}$ still depend on $N$ in our bounds (2.13) and (2.15). Our next task is to relate these energies to the NLS ground state energy $e_{\mathrm{nls}}$. In general, $e_{\mathrm{H}}$ will not converge to $e_{\mathrm{nls}}$. For this to be true, some stability properties of the interaction potential $w$ are needed, further discussed in the following.

2.2.1. Stability properties of $w$. An even potential $W$ is called classically stable [51] when

$$
\sum_{1 \leq i<j \leq N} W\left(x_{i}-x_{j}\right) \geq-C N, \quad \forall x_{1}, \ldots, x_{N} \in \mathbb{R}^{d}, \forall N \geq 2 .
$$

By integrating against a factorized measure $\rho\left(x_{1}\right) \cdots \rho\left(x_{N}\right)$ and letting $N \rightarrow \infty$, we see that (2.19) implies

$$
\iint_{\mathbb{R}^{d} \times \mathbb{R}^{d}} \rho(x) \rho(y) W(x-y) d x d y \geq 0, \quad \forall \rho \geq 0 .
$$

Conversely, when $W$ is bounded, (2.20) implies (2.19) as is seen by taking $\rho=\sum_{i=1}^{N} \delta_{x_{i}}$. By dilating $\rho$ one can see that (2.20) implies $\int_{\mathbb{R}^{d}} W \geq 0$.

The relevance of classical stability to our quantum problem depends on the dimension: - When $d \geq 3$, the nonlinear term of the NLS functional is super-critical with respect to the kinetic energy and we shall take (2.20) to be our assumption. Note that it is really necessary to ensure that $e_{\mathrm{H}}$ does not converge to $-\infty$, which can be seen immediately by

\footnotetext{
$3 \widehat{w}$ denotes the Fourier transform of $w$.
} 
taking a trial state $u_{N}(x)=N^{d \beta / 2} u\left(N^{\beta} x\right)$. In other words, in 3D, stability of the quantum problem requires classical stability.

- In dimension $d=2$, the nonlinear term of the NLS functional is critical with respect to the kinetic energy. Classical stability is then not the optimal concept and Condition (2.20) can be relaxed a bit with the help of the kinetic energy. We say that an even potential $W$ is Hartree-stable when

$$
\|u\|_{L^{2}}^{2}\|\nabla u\|_{L^{2}}^{2}+\frac{1}{2} \iint_{\mathbb{R}^{2} \times \mathbb{R}^{2}}|u(x)|^{2}|u(y)|^{2} W(x-y) d x d y \geq 0
$$

for all $u \in H^{1}\left(\mathbb{R}^{2}\right)$. Clearly, a classically-stable potential is also Hartree-stable. Replacing $u$ by $\lambda u(\lambda x)$ and taking the limit $\lambda \rightarrow 0$, we see that such a potential $W$ must satisfy

$$
\|u\|_{L^{2}}^{2}\|\nabla u\|_{L^{2}}^{2}+\frac{1}{2}\left(\int_{\mathbb{R}^{2}} W\right) \int_{\mathbb{R}^{2}}|u(x)|^{4} d x \geq 0, \quad \forall u \in H^{1}\left(\mathbb{R}^{2}\right) .
$$

This exactly means that

$$
\int_{\mathbb{R}^{2}} W(x) d x \geq-a^{*}
$$

where $a^{*}$ is the critical interaction strength [58, 60, 27, 43] for existence of a ground state for the NLS functional, that is, $a^{*}:=\|Q\|_{L^{2}\left(\mathbb{R}^{2}\right)}^{2}$, where $Q \in H^{1}\left(\mathbb{R}^{2}\right)$ is the unique (up to translations) positive radial solution of

$$
-\Delta Q+Q-Q^{3}=0 .
$$

On the other hand, using the Cauchy-Schwarz and Young's inequalitiest we have

$\iint_{\mathbb{R}^{2} \times \mathbb{R}^{2}}|u(x)|^{2}|u(y)|^{2} W(x-y) d x d y \geq-\left\|u^{2}\right\|_{L^{2}}\left\||u|^{2} * W^{-}\right\|_{L^{2}} \geq-\left(\int_{\mathbb{R}^{2}} W^{-}\right) \int_{\mathbb{R}^{2}}|u(x)|^{4} d x$, and we see that $\int_{\mathbb{R}^{2}} W^{-} \leq a^{*}$ implies (2.21). In the following we shall actually need a slightly stronger notion of Hartree-stability, obtained by requiring (2.21) with a strict inequality,

$$
\inf _{u \in H^{1}\left(\mathbb{R}^{2}\right)}\left(\frac{\iint_{\mathbb{R}^{2} \times \mathbb{R}^{2}}|u(x)|^{2}|u(y)|^{2} W(x-y) d x d y}{2\|u\|_{L^{2}\left(\mathbb{R}^{2}\right)}^{2}\|\nabla u\|_{L^{2}\left(\mathbb{R}^{2}\right)}^{2}}\right)>-1
$$

which plays the same role as the assumption $\int_{\mathbb{R}^{2}} W>-a^{*}$ in the NLS case.

- In dimension $d=1$, the NLS nonlinearity is subcritical and the quantum system is always stable thanks to the kinetic energy, whence the absence of specific assumptions in this case.

In order to simplify our presentation, from now on we use the word "stable" for a potential $W$ that satisfies (2.23) in dimension $d=2$ and (2.20) in dimension $d \geq 3$. The importance of these concepts is illustrated in the following.

Proposition 2.3 (Convergence of $e_{\mathrm{H}}$ towards $e_{\mathrm{nls}}$ ).

Let $\beta>0$. We assume that (2.1), (2.2) and (2.3) hold true, and further suppose that $|x| w(x) \in L^{1}\left(\mathbb{R}^{d}\right)$.

- If $d=1$, or $d=2,3$ with $w$ stable, then we have

$$
\left|e_{\mathrm{H}}-e_{\mathrm{nls}}\right| \leq C N^{-\beta} \text {. }
$$

\footnotetext{
${ }^{4}$ with $W^{-}:=-\min (0, W)$ the negative part of $W$
} 
Furthermore, minimizers for $e_{\mathrm{H}}$ converge in the limit $N \rightarrow \infty$ to a minimizer for $e_{\mathrm{nl}}$, after extraction of a subsequence.

- If $d=2$ and

$$
\inf _{u \in H^{1}\left(\mathbb{R}^{2}\right)}\left(\frac{\iint_{\mathbb{R}^{2} \times \mathbb{R}^{2}}|u(x)|^{2}|u(y)|^{2} w(x-y) d x d y}{2\|u\|_{L^{2}\left(\mathbb{R}^{2}\right)}^{2}\|\nabla u\|_{L^{2}\left(\mathbb{R}^{2}\right)}^{2}}\right)<-1,
$$

or if $d \geq 3$ and $w$ is not classically stable (in the sense of (2.20) ), then

$$
\lim _{N \rightarrow \infty} e_{\mathrm{H}}=-\infty .
$$

The decay condition $|x| w(x) \in L^{1}$ is a technical assumption which allows us to obtain (2.24) by controling the error induced by replacing the interaction potential $w_{N}$ in the Hartree model with the delta-potential in the NLS model. Without this assumption we still have $e_{\mathrm{H}} \rightarrow e_{\mathrm{nls}}$ but with no estimate on the convergence rate. Of course (2.26) immediately implies

$$
\lim _{N \rightarrow \infty} \frac{E(N)}{N}=-\infty
$$

since $E(N) \leq N e_{\mathrm{H}}$. In the above result, the stability condition on $w$ is optimal in dimension $d=3$. If $w$ is classically unstable we have (2.27) but it could be that $\int_{\mathbb{R}^{3}} w \geq 0$, and then $e_{\mathrm{nls}}$ is finite and therefore cannot be related to the limit of the $N$-body problem. In dimension $d=2$ we are only missing the case of equality in (2.23) and the stability condition is therefore nearly optimal.

2.2.2. Convergence of the many-body problem. With Theorem 2.1 and Proposition 2.3 at hand, it is an easy task to deduce error bounds between $E(N) / N$ and the NLS minimum energy.

Theorem 2.4 (Derivation of the NLS ground state energy).

Let $\beta>0$. We assume that (2.1), (2.2) and (2.3) hold true, and further suppose that $|x| w(x) \in L^{1}\left(\mathbb{R}^{d}\right)$.

- If $d=1$, then we have

$$
C N^{-\beta}+e_{\mathrm{nls}} \geq \frac{E(N)}{N} \geq e_{\mathrm{nls}}-C N^{-\beta}-C N^{-\frac{1}{4+2 / s}}
$$

for all $\beta>0$.

- If $d=2$ and $w$ is stable (in the sense of (2.23)), or if $d=3$ and $w-\eta|w|$ is stable (in the sense of (2.20) ) for some $0<\eta<1$, then

$$
e_{\mathrm{nls}}+C N^{-\beta} \geq \frac{E(N)}{N} \geq e_{\mathrm{nls}}-C N^{-\beta}-C N^{-\frac{1-d \beta(1+d / 2+d / s)}{2+d / s+d / 2}}
$$

provided that $\beta$ satisfies (2.14).

- Finally, if $d=3$ and $w$ is stable (in the sense of (2.20) ), then we have

$$
e_{\mathrm{nls}}+C N^{-\beta} \geq \frac{E(N)}{N} \geq e_{\mathrm{nls}}-C N^{-\beta}-C N^{d \beta-\frac{1}{2+d / s+d / 2}}
$$

provided that $\beta$ satisfies (2.16). 
We remark that when $d=2$, since the stability assumption (2.23) is strict, the stability for $w$ implies the stability for $w-\eta|w|$ for some $0<\eta<1$. However, the same does not hold when $d=3$ and the error estimate (2.29) is really better than (2.30) (and similarly for the range of $\beta$ ).

The proofs of Theorems 2.1 and 2.4 also imply some estimates on the ground states themselves. In the absence of any assumption on the behavior of the Hartree functional we cannot convert them into convergence rates for the states. In the NLS limit we nevertheless obtain convergence of states and Bose-Einstein condensation:

Theorem 2.5 (Convergence of states in the NLS limit).

We use the same assumptions as in Theorem 2.4. Denote $\Psi_{N}$ a ground state of the manybody Hamiltonian (1.4) and

$$
\gamma_{N}^{(n)}:=\operatorname{Tr}_{n+1 \rightarrow N}\left|\Psi_{N}\right\rangle\left\langle\Psi_{N}\right|
$$

its n-body reduced density matrix. We have, modulo a subsequence,

$$
\lim _{N \rightarrow \infty} \gamma_{N}^{(n)}=\int_{u \in \mathcal{M}_{\mathrm{nls}}} d \mu(u)\left|u^{\otimes n}\right\rangle\left\langle u^{\otimes n}\right|
$$

strongly in the trace-class for any $n \geq 1$, with $\mu$ a Borel probability measure supported on

$$
\mathcal{M}_{\mathrm{nls}}=\left\{u \in L^{2}\left(\mathbb{R}^{d}\right),\|u\|_{L^{2}}=1, \mathcal{E}_{\mathrm{nls}}[u]=e_{\mathrm{nls}}\right\} .
$$

In particular, when the NLS ground state $u_{\mathrm{nls}}$ is unique (modulo a constant phase), we have convergence for the whole sequence

$$
\lim _{N \rightarrow \infty} \gamma_{N}^{(n)}=\left|u_{\mathrm{nls}}^{\otimes n}\right\rangle\left\langle u_{\mathrm{nls}}^{\otimes n}\right| \text {, strongly in trace-class norm. }
$$

Uniqueness of $u_{\text {nls }}$ is ensured when $\int w$ is either $\geq 0$ or small enough in absolute value in dimensions $d=1,2$, and the vector potential $A$ is small enough. Loss of uniqueness does occur if these assumptions are not satisfied, which is intimately linked to symmetry breaking phenomena [3, 5, 16, 17, 27, 52. When the NLS functional satisfies some stability properties one may obtain error estimates on states, see Remark 4.2.

The main virtue of the estimates of Theorems 2.1 and 2.4 is that they do not depend on special properties of the interaction potentials (apart from the necessary stability conditions). They actually also do not depend on the vector potential $A$ and they would be exactly the same if the one-body term in the many-particle Hamiltonian is perturbed by any bounded operator. As we have said, there is however no reason to think that the rates of convergence are optimal.

When $d \geq 2$, the estimates of Theorem 2.1 deteriorate with increasing $\beta$ and this results in a decrease of the range of applicability to the NLS limit. For the typical settings described above we obtain the desired result

$$
\frac{E(N)}{N} \rightarrow e_{\mathrm{nls}} \text { when } N \rightarrow \infty
$$

under the conditions listed in Table 2. In 3D we distinguish the case where $w-\eta|w|$ is stable for some small fixed $\eta$ where we obtain better estimates than when $w$ is simply stable. In the former case we say that $w$ is $\eta$-stable. It remains an open problem to improve the convergence rates we obtain and to generalize the results to larger values of $\beta$. In 
particular, we expect that one should obtain the same NLS limit for all $0<\beta<1$ when $d \geq 2$, see [38, 39] for the repulsive case.

\begin{tabular}{|r|c|c||c|}
\hline & $d=3, w$ stable & $d=3, w \eta$-stable & $d=2$ \\
\hline$s=2$ & $\beta<1 / 15$ & $\beta<1 / 12$ & $\beta<1 / 6$ \\
\hline$s=\infty$ & $\beta<2 / 21$ & $\beta<2 / 15$ & $\beta<1 / 4$ \\
\hline
\end{tabular}

TABLE 2. Maximal value of $\beta$ in the NLS limit $(\beta>0)$.

The rest of the paper is organized as follows: the core of the analysis is in Section 3 where we prove Theorem 2.1. Next we turn to the proof of Proposition 2.3 in Section 4 where we also conclude the proofs of Theorems 2.4 and 2.5 .

\section{ERror bounds For Hartree theORY}

In this section, we prove Theorem 2.1.

3.1. Quantum de Finetti and localization. Let $\gamma_{N}$ be an arbitrary (mixed) state in the bosonic Hilbert space $\mathfrak{H}^{N}=\bigotimes_{s}^{N} \mathfrak{H}$, i.e. a positive operator satisfying $\operatorname{Tr} \gamma_{N}=1$. For every $k=1,2, \ldots, N$, the $k$-particle density matrix $\gamma_{N}^{(k)}$ is obtained by taking the partial trace over all but the first $k$ variables:

$$
\gamma_{N}^{(k)}=\operatorname{Tr}_{k+1 \rightarrow N}\left[\gamma_{N}\right]
$$

One of the main advantages of the reduced density matrices is that we can write

$$
\frac{E(N)}{N}=\frac{1}{N}\left\langle\Psi_{N}, H_{N} \Psi_{N}\right\rangle=\frac{1}{2} \operatorname{Tr}_{\mathfrak{H}^{2}}\left[H_{2} \gamma_{N}^{(2)}\right]
$$

where $\gamma_{N}=\left|\Psi_{N}\right\rangle\left\langle\Psi_{N}\right|$ and

$$
H_{2}=-\left(\nabla_{x_{1}}+i A\left(x_{1}\right)\right)^{2}+V\left(x_{1}\right)-\left(\nabla_{x_{2}}+i A\left(x_{2}\right)\right)^{2}+V\left(x_{2}\right)+N^{d \beta} w\left(N^{\beta}\left(x_{1}-x_{2}\right)\right) .
$$

When $\beta=0, H_{2}$ is independent of $N$ and the limit $E(N) / N \rightarrow e_{\mathrm{H}}$ essentially comes from the structure of $\gamma_{N}^{(2)}$ in the large $N$ limit. If one does not need an error estimate, this convergence follows easily from a compactness argument and the quantum de Finetti theorem [57, 29]. This is explained in [32, Section 3].

When $\beta>0$, we have to deal with a $N$-dependent interaction potential and the compactness argument in [32] is not sufficient. Our strategy in this paper is to use a localization method to reduce the problem to a finite dimensional setting. We may then employ the following quantitative version of the quantum de Finetti theorem, originally proved in 12 . (see [11, 28, 31] for variants of the proof and 22] for an earlier result in this direction):

\section{Theorem 3.1 (Quantitative quantum de Finetti in finite dimension).}

Let $\mathfrak{K}$ be a finite dimensional Hilbert space. For every state $G_{N}$ on $\mathfrak{K}^{N}:=\bigotimes_{s}^{N} \mathfrak{K}$ and for every $k=1,2, \ldots, N$ we have

$$
\left.\operatorname{Tr}_{\mathfrak{K}^{n}}\left|G_{N}^{(k)}-\int_{S \mathfrak{K}}\right| u^{\otimes k}\right\rangle\left\langle u^{\otimes k}\left|d \mu_{G_{N}}(u)\right| \leq \frac{4 k \operatorname{dim} \mathfrak{K}}{N}\right.
$$

where

$$
G_{N}^{(n)}:=\operatorname{Tr}_{n+1 \rightarrow N}\left[G_{N}\right]
$$


and

$$
d \mu_{G_{N}}(u):=\operatorname{dim} \mathfrak{K}^{N}\left\langle u^{\otimes N}, G_{N} u^{\otimes N}\right\rangle d u
$$

with du being the normalized uniform (Haar) measure on the unit sphere $S \mathfrak{K}$.

Remark 3.2. The measure $\mu_{G_{N}}$ is a probability measure thanks to Schur's formula

$$
\mathbb{1}_{\mathfrak{K}^{N}}=\operatorname{dim} \mathfrak{K}^{N} \int_{S \mathfrak{K}}\left|u^{\otimes k}\right\rangle\left\langle u^{\otimes k}\right| d u .
$$

We shall not need the explicit expression (3.3). If one could find a different construction giving a better error estimate, the convergence rates of our main theorems would be improved using the method we describe below.

We will apply Theorem 3.1 to the low-lying energy subspace of the (magnetic) Schrödinger operator $H_{1}=-(\nabla+i A)^{2}+V$ acting on $\mathfrak{H}=L^{2}\left(\mathbb{R}^{d}\right)$. We denote by $P_{-}$and $P_{+}$the spectral projectors above and below the energy cut-off $L$ :

$$
P_{-}=\mathbb{1}_{(-\infty, L)}\left(H_{1}\right), P_{+}=\mathbb{1}_{\mathfrak{H}}-P_{-}=P_{-}^{\perp} .
$$

Thanks to our assumption (2.2) and (2.3), the dimension of the low-lying subspace

$$
N_{L}:=\operatorname{dim}\left(P_{-} \mathfrak{H}\right)=\text { number of eigenvalues of } H_{1} \text { below } L
$$

is finite. Moreover it is controlled by a semi-classical inequality "à la Cwikel-Lieb-Rosenblum", stated in the next lemma. We refer to [36, Chapter 4] for a thorough discussion of related inequalities.

Lemma 3.3 (Low-lying bound states of the one-body Hamiltonian). Let $V$ and $A$ satisfy (2.2) and (2.3), respectively. Then for $L$ large enough we have

$$
N_{L} \leq C L^{d / s+d / 2} .
$$

Proof. The number of eigenvalues of $(-i \nabla+A)^{2}+V$ below $L$ can be estimated by

$$
N_{L} \leq \operatorname{Tr}_{L^{2}\left(\mathbb{R}^{d}\right)}\left[\exp \left(-\frac{(-i \nabla+A)^{2}+V-L}{L}\right)\right] \leq \frac{1}{(2 \pi)^{d}} \iint_{\mathbb{R}^{d} \times \mathbb{R}^{d}} e^{-\frac{|p|^{2}+V-L}{L}} d x d p,
$$

using [13, Thm. 2.1] and [55, Thm 15.8]. Using our assumption (2.2) that $V(x) \geq c|x|^{s}-C$ and changing variables gives the result.

We will combine the de Finetti theorem and the localization method in Fock space, which provides the correct way of restricting a quantum $N$-body state to a subspace of $\mathfrak{H}$. Let us quickly recall this procedure, following the notation of [30].

Let $\gamma_{N}$ be an arbitrary $N$-body (mixed) state. With the given projections $P_{-}, P_{+}$, there are localized states $G_{N}^{-}, G_{N}^{+}$in the Fock space

$$
\mathcal{F}(\mathfrak{H})=\mathbb{C} \oplus \mathfrak{H} \oplus \mathfrak{H}^{2} \oplus \cdots
$$

of the form

$$
G_{N}^{ \pm}=G_{N, 0}^{ \pm} \oplus G_{N, 1}^{ \pm} \oplus \cdots \oplus G_{N, N}^{ \pm} \oplus 0 \oplus \cdots
$$

with the crucial property that their reduced density matrices satisfy

$$
P_{ \pm}^{\otimes n} \gamma_{N}^{(n)} P_{ \pm}^{\otimes n}=\left(G_{N}^{ \pm}\right)^{(n)}=\left(\begin{array}{c}
N \\
n
\end{array}\right)^{-1} \sum_{k=n}^{N}\left(\begin{array}{l}
k \\
n
\end{array}\right) \operatorname{Tr}_{n+1 \rightarrow k}\left[G_{N, k}^{ \pm}\right]
$$


for any $0 \leq n \leq N$. Here we use the convention that

$$
\gamma_{N}^{(n)}:=\operatorname{Tr}_{n+1 \rightarrow N}\left[\gamma_{N}\right]
$$

which differs from the convention of [30], whence the different numerical factors in (3.8).

The relations (3.8) determine the localized states $G_{N}^{-}, G_{N}^{+}$uniquely and they ensure that $G_{N}^{-}$and $G_{N}^{+}$are (mixed) states on the Fock spaces $\mathcal{F}\left(P_{-} \mathfrak{H}\right)$ and $\mathcal{F}\left(P_{+} \mathfrak{H}\right)$, respectively:

$$
\sum_{k=0}^{N} \operatorname{Tr}\left[G_{N, k}^{-}\right]=\sum_{k=0}^{N} \operatorname{Tr}\left[G_{N, k}^{+}\right]=1
$$

Due to (3.1), we are mainly interested in the two-particle density matrices. Applying the quantitative de Finetti Theorem 3.1 to the localized state $G_{N}^{-}$, we obtain the following

Lemma 3.4 (Quantitative quantum de Finetti for the localized state.). Let $\gamma_{N}$ be an arbitrary $N$-body (mixed) state and for every $L>0$, we have

$$
\left.\operatorname{Tr}_{\mathfrak{H}^{2}}\left|P_{-}^{\otimes 2} \gamma_{N}^{(2)} P_{-}^{\otimes 2}-\int_{S P_{-} \mathfrak{H}}\right| u^{\otimes 2}\right\rangle\left\langle u^{\otimes 2}\left|d \mu_{N}(u)\right| \leq \frac{8 N_{L}}{N}\right.
$$

where

$$
d \mu_{N}(u)=\sum_{k=2}^{N} \frac{k(k-1)}{N(N-1)} d \mu_{N, k}(u), \quad d \mu_{N, k}(u)=\operatorname{dim}\left(P_{-} \mathfrak{H}\right)_{s}^{k}\left\langle u^{\otimes k}, G_{N, k}^{-} u^{\otimes k}\right\rangle d u .
$$

Proof. Applying the quantitative de Finetti Theorem 3.1 we have

$$
\left.\operatorname{Tr}_{\mathfrak{H}^{2}}\left|\operatorname{Tr}_{3 \rightarrow k}\left[G_{N, k}^{-}\right]-\int_{S P_{-} \mathfrak{H}}\right| u^{\otimes 2}\right\rangle\left\langle u^{\otimes 2}\left|d \mu_{N, k}(u)\right| \leq 8 \frac{N_{L}}{k} \operatorname{Tr}_{\mathfrak{H}^{k}}\left[G_{N, k}^{-}\right]\right.
$$

where $d \mu_{N, k}(u)=\operatorname{dim}\left(P_{-} \mathfrak{H}\right)_{s}^{k}\left\langle u^{\otimes k}, G_{N, k}^{-} u^{\otimes k}\right\rangle d u$. Combining this and (3.8) we get

$$
\begin{aligned}
\left.\operatorname{Tr}_{\mathfrak{H}^{2}}\left|P_{-}^{\otimes 2} \gamma_{N}^{(2)} P_{-}^{\otimes 2}-\int_{S P_{-} \mathfrak{H}}\right| u^{\otimes 2}\right\rangle\left\langle u^{\otimes 2}\left|d \mu_{N}(u)\right|\right. & \leq \sum_{k=2}^{N}\left(\begin{array}{c}
N \\
2
\end{array}\right)^{-1}\left(\begin{array}{c}
k \\
2
\end{array}\right) \frac{8 N_{L}}{k} \operatorname{Tr}_{\mathfrak{H}^{k}}\left[G_{N, k}^{-}\right] \\
& =\frac{8 N_{L}}{N} \sum_{k=2}^{N} \frac{k-1}{N-1} \operatorname{Tr}_{\mathfrak{H}^{k}}\left[G_{N, k}^{-}\right] \leq \frac{8 N_{L}}{N},
\end{aligned}
$$

since $\sum_{k=0}^{N} \operatorname{Tr}_{\mathfrak{H}^{k}}\left[G_{N, k}^{-}\right]=1$.

Remark 3.5 (De Finetti measure for the $n$-body reduced density matrix).

We will later apply the same idea to any $n$-body reduced density matrix of $\gamma_{N}$ with $n \geq 2$. The de Finetti measures obtained in this way, namely

$$
d \mu_{N}^{n}(u)=\sum_{k=n}^{N}\left(\begin{array}{l}
N \\
n
\end{array}\right)^{-1}\left(\begin{array}{l}
k \\
n
\end{array}\right) d \mu_{N, k}(u)=\sum_{k=n}^{N} \frac{k(k-1) \ldots(k-n+1)}{N(N-1) \ldots(N-n+1)} d \mu_{N, k}(u),
$$

a priori depend on $n$, unless $G_{N, k}^{-}=0$ for any $k=0 \ldots N-1$, namely unless all the particles are $P_{-}$localized. However, in some situations (e.g. in our case in Section 4.3), we can show that most particles are $P_{-}$localized, and thus all these $n$-dependent measures actually converge to the same limit. 
We shall prove Theorem 2.1 in the following subsections. The core of the proof is in Section 3.3, where Lemma 3.4 plays an essential role, with some preparation in Section 3.2 and some final computations in Section 3.4 .

3.2. Truncated two-body Hamiltonian. Recall from (3.1) that

$$
\frac{E(N)}{N}=\frac{1}{2} \operatorname{Tr}\left[H_{2} \gamma_{N}^{(2)}\right]
$$

with $\gamma_{N}=\left|\Psi_{N}\right\rangle\left\langle\Psi_{N}\right|$. In order to reduce to a finite dimensional setting, we will replace the Hamiltonian $H_{2}$ by the localized operator $P_{-} \otimes P_{-} H_{2} P_{-} \otimes P_{-}$, up to a small modification of the interaction potential $w_{N}$. The crucial fact is that if the energy cut-off $L$ is large enough (in comparison with $\left\|w_{N}\right\|_{L^{\infty}}$ ), then the kinetic energy of the $P_{+}$localized particles can be used to control both the localization error and the number of $P_{+}$localized particles.

For convenience we introduce a two-particle operator with modified interaction:

$$
\begin{aligned}
H_{2}^{\varepsilon}=-\left(\nabla_{x_{1}}-i A\left(x_{1}\right)\right)^{2}+V\left(x_{1}\right)-\left(\nabla_{x_{2}}-i A\left(x_{2}\right)\right)^{2}+V\left(x_{2}\right) & \\
& +w_{N}\left(x_{1}-x_{2}\right)-\varepsilon\left|w_{N}\left(x_{1}-x_{2}\right)\right| .
\end{aligned}
$$

An important tool is then the following.

\section{Lemma 3.6 (Truncated two-body Hamiltonian).}

Assuming that $0<\varepsilon \leq 1$ and $L \geq C N^{d \beta} \varepsilon^{-1}$ (resp. $L \geq C \varepsilon^{-2}$ if $d=1$ and $\beta>0$ ) for a large enough constant $C$, we have

$$
H_{2} \geq P_{-}^{\otimes 2} H_{2}^{\varepsilon} P_{-}^{\otimes 2}+\frac{L}{2}\left(P_{+} H_{1} P_{+} \otimes \mathbb{1}+\mathbb{1} \otimes P_{+} H_{1} P_{+}\right) .
$$

The estimates in the $d=1$ case use a simple lemma that we prove at the end of this subsection:

Lemma 3.7 (Sobolev-type inequality in dimension $d=1$ ).

For any even potential $W \in L^{1}(\mathbb{R})$, we have in dimension $d=1$

$$
H_{1} \otimes \mathbb{1}+\mathbb{1} \otimes H_{1}+W(x-y) \geq-C\left(\int_{\mathbb{R}} W^{-}\right)^{2}-C .
$$

Proof of Lemma 3.6. For the two-body non-interacting Hamiltonian

$$
H_{1} \otimes \mathbb{1}+\mathbb{1} \otimes H_{1}=-\left(\nabla_{x_{1}}-i A\left(x_{1}\right)\right)^{2}+V\left(x_{1}\right)-\left(\nabla_{x_{2}}-i A\left(x_{2}\right)\right)^{2}+V\left(x_{2}\right),
$$

we use that

$$
H_{1}=P_{-} H_{1} P_{-}+P_{+} H_{1} P_{+}
$$

and obtain

$$
\begin{aligned}
H_{1} \otimes \mathbb{1}+\mathbb{1} \otimes H_{1}= & P_{-} H_{1} P_{-} \otimes \mathbb{1}+\mathbb{1} \otimes P_{-} H_{1} P_{-}+P_{+} H_{1} P_{+} \otimes \mathbb{1}+\mathbb{1} \otimes P_{+} H_{1} P_{+} \\
= & \left(P_{-}\right)^{\otimes 2}\left(H_{1} \otimes \mathbb{1}+\mathbb{1} \otimes H_{1}\right)\left(P_{-}\right)^{\otimes 2}+P_{-} H_{1} P_{-} \otimes P_{+} \\
& +P_{+} \otimes P_{-} H_{1} P_{-}+P_{+} H_{1} P_{+} \otimes \mathbb{1}+\mathbb{1} \otimes P_{+} H_{1} P_{+} .
\end{aligned}
$$

Since $H_{1}$ is bounded from below, we have $P_{-} H_{1} P_{-} \geq-C P_{-} \geq-C$ and we obtain $H_{1} \otimes \mathbb{1}+\mathbb{1} \otimes H_{1} \geq\left(P_{-}\right)^{\otimes 2}\left(H_{1} \otimes \mathbb{1}+\mathbb{1} \otimes H_{1}\right)\left(P_{-}\right)^{\otimes 2}+P_{+}\left(H_{1}-C\right) P_{+} \otimes \mathbb{1}+\mathbb{1} \otimes P_{+}\left(H_{1}-C\right) P_{+}$. 
We now consider the interaction term and use the shorthand notation $w_{N}$ for the twobody multiplication operator $\left(x_{1}, x_{2}\right) \mapsto w_{N}\left(x_{1}-x_{2}\right)$. We write again

$$
\begin{aligned}
w_{N} & =\left(P_{-}+P_{+}\right)^{\otimes 2} w_{N}\left(P_{-}+P_{+}\right)^{\otimes 2} \\
& =\left(P_{-}\right)^{\otimes 2} w_{N}\left(P_{-}\right)^{\otimes 2}+\left(P_{-}\right)^{\otimes 2} w_{N} \Pi+\Pi w_{N}\left(P_{-}\right)^{\otimes 2}+\Pi w_{N} \Pi,
\end{aligned}
$$

with the orthogonal projection $\Pi:=P_{-} \otimes P_{+}+P_{+} \otimes P_{-}+P_{+} \otimes P_{+}$. To bound the error terms we use the inequality

$$
P A Q+Q A P \geq-\varepsilon P|A| P-\varepsilon^{-1} Q|A| Q,
$$

valid for any self-adjoint operator $A$, and any orthogonal projectors $P, Q$. If $A$ is positive this follows by writing

$$
\left(\varepsilon^{1 / 2} P \pm \varepsilon^{-1 / 2} Q\right) A\left(\varepsilon^{1 / 2} P \pm \varepsilon^{-1 / 2} Q\right) \geq 0
$$

and the general case is obtained by using the same bound applied to $A^{+}$and $A^{-}$separately. We thereby deduce that

$$
\left(P_{-}\right)^{\otimes 2} w_{N} \Pi+\Pi w_{N}\left(P_{-}\right)^{\otimes 2} \geq-\varepsilon\left(P_{-}\right)^{\otimes 2}\left|w_{N}\right|\left(P_{-}\right)^{\otimes 2}-\varepsilon^{-1} \Pi\left|w_{N}\right| \Pi
$$

and, therefore,

$$
w_{N} \geq\left(P_{-}\right)^{\otimes 2}\left(w_{N}-\varepsilon\left|w_{N}\right|\right)\left(P_{-}\right)^{\otimes 2}-\left(1+\varepsilon^{-1}\right) \Pi\left|w_{N}\right| \Pi .
$$

Using now $\left|w_{N}\right| \leq C N^{d \beta}$ and collecting our estimates we find

$$
\begin{aligned}
H_{2} & \geq\left(P_{-}\right)^{\otimes 2} H_{2}^{\varepsilon}\left(P_{-}\right)^{\otimes 2}+P_{+}\left(H_{1}-C\right) P_{+} \otimes \mathbb{1}+\mathbb{1} \otimes P_{+}\left(H_{1}-C\right) P_{+}-C\left(1+\varepsilon^{-1}\right) N^{d \beta} \Pi \\
& \geq\left(P_{-}\right)^{\otimes 2} H_{2}^{\varepsilon}\left(P_{-}\right)^{\otimes 2}+\frac{1}{2}\left(P_{+} H_{1} P_{+} \otimes \mathbb{1}+\mathbb{1} \otimes P_{+} H_{1} P_{+}\right)+\left(\frac{L}{2}-C-C\left(1+\varepsilon^{-1}\right) N^{d \beta}\right) \Pi
\end{aligned}
$$

and the result follows in dimensions $d \geq 2$ or when $\beta=0$ and $d=1$.

When $d=1$ and $\beta>0$, we may come back to (3.16) and use Lemma 3.7 to obtain

$$
\begin{aligned}
H_{2} \geq\left(P_{-}\right)^{\otimes 2} H_{2}^{\varepsilon}\left(P_{-}\right)^{\otimes 2}+\frac{1}{2}\left(P_{+} H_{1} P_{+} \otimes \mathbb{1}+\mathbb{1} \otimes P_{+} H_{1} P_{+}\right) \\
+\Pi\left(L / 4-C+\left(H_{1} \otimes \mathbb{1}+\mathbb{1} \otimes H_{1}\right) / 4-\left(1+\varepsilon^{-1}\right)\left|w_{N}\right|\right) \Pi \\
\geq\left(P_{-}\right)^{\otimes 2} H_{2}^{\varepsilon}\left(P_{-}\right)^{\otimes 2}+\frac{1}{2}\left(P_{+} H_{1} P_{+} \otimes \mathbb{1}+\mathbb{1} \otimes P_{+} H_{1} P_{+}\right)+\Pi\left(L / 4-C-C \varepsilon^{-2}\right) \Pi .
\end{aligned}
$$

Proof of Lemma 3.7. In 1D we can bound

$$
\int_{\mathbb{R}}\left|W(x)\left\|\left.u(x)\right|^{2} \leq\left(\int_{\mathbb{R}}|W|\right)\right\| u\left\|_{L^{\infty}(\mathbb{R})}^{2} \leq C\right\| W\left\|_{L^{1}(\mathbb{R})}\right\| u\left\|_{L^{2}(\mathbb{R})}\right\| u^{\prime} \|_{L^{2}(\mathbb{R})}\right.
$$

for every $u \in H^{1}(\mathbb{R})$. We conclude that

$$
-d^{2} / d x^{2}+W \geq-d^{2} / d x^{2}-W^{-} \geq-C\left\|W^{-}\right\|_{L^{1}(\mathbb{R})}^{2}-C .
$$

Using the pointwise diamagnetic inequality $|(\nabla+i A) u| \geq|\nabla| u||$ and removing the center of mass, the estimate is similar for the two-particle operator.

\footnotetext{
${ }^{5}$ We copy the proof that the diagonal part of a positive hermitian matrix controls the off-diagonal part.
} 
3.3. Bound on the localized energy. Now we turn to the main step of the proof of Theorem 2.1] we compare the energy corresponding to the localized operator in the right side of (3.12) with the Hartree energy $e_{\mathrm{H}}$.

Let $\gamma_{N}=\left|\Psi_{N}\right\rangle\left\langle\Psi_{N}\right|$ be the density matrix of a ground state $\Psi_{N} \in \mathfrak{H}^{N}$ of the $N$-body Hamiltonian $H_{N}$ (known to exist in our setting of trapped systems). We have

\section{Lemma 3.8 (Lower bound to the localized energy).}

For $0<\varepsilon \leq 1, L \geq C N^{d \beta} \varepsilon^{-1}$ (resp. $L \geq C \varepsilon^{-2}$ if $d=1$ and $\beta>0$ ) and $N$ large enough, we have

$$
\frac{1}{2} \operatorname{Tr}\left[P_{-}^{\otimes 2} H_{2}^{\varepsilon} P_{-}^{\otimes 2} \gamma_{N}^{(2)}\right]+\frac{L}{4} \operatorname{Tr}\left[P_{+} \gamma_{N}^{(1)}\right] \geq e_{\mathrm{H}}^{\varepsilon}-\frac{C L^{1+d / s+d / 2}}{N} .
$$

Proof. We will employ the same notations for localized states $G_{N}^{ \pm}$obtained from the $N$-body state $\gamma_{N}$ and the projections $P_{ \pm}$as in Subsection 3.1. By Lemma 3.4, we have

$$
\left.\operatorname{Tr}_{\mathfrak{H}^{2}}\left|P_{-}^{\otimes 2} \gamma_{N}^{(2)} P_{-}^{\otimes 2}-\int_{S P_{-} \mathfrak{H}}\right| u^{\otimes 2}\right\rangle\left\langle u^{\otimes 2}\left|d \mu_{N}(u)\right| \leq \frac{8 N_{L}}{N}\right.
$$

with $d \mu_{N}$ defined as in (3.10). On the other hand,

$$
\left\|P_{-}^{\otimes 2} H_{2}^{\varepsilon} P_{-}^{\otimes 2}\right\| \leq 2 L+(1+\varepsilon)\left\|w_{N}\right\|_{L^{\infty}} \leq C L
$$

in operator norm because we have truncated the high energy spectrum of the one-body part and the two-body potential is bounded by $\left\|w_{N}\right\|_{L^{\infty}} \leq C N^{d \beta} \leq C L$. In dimension $d=1$, the estimate is the same but we use that $\left|w_{N}\right| \leq H_{1} \otimes \mathbb{1}+\mathbb{1} \otimes H_{1}+C$, by Lemma 3.7. Therefore,

$$
\begin{aligned}
\frac{1}{2} \operatorname{Tr}\left[P_{-}^{\otimes 2} H_{2}^{\varepsilon} P_{-}^{\otimes 2} \gamma_{N}^{(2)}\right] & \geq \frac{1}{2} \int_{S P_{-} \mathfrak{H}} \operatorname{Tr}_{\mathfrak{H}^{2}}\left[H_{2}^{\varepsilon}\left|u^{\otimes 2}\right\rangle\left\langle u^{\otimes 2}\right|\right] d \mu_{N}-\frac{C L N_{L}}{N} \\
& \geq \int_{S P_{-} \mathfrak{H}} \mathcal{E}_{\mathrm{H}}^{\varepsilon}[u] d \mu_{N}-\frac{C L^{1+d / s+d / 2}}{N} \\
& \geq \int_{S P_{-} \mathfrak{H}}\left(\mathcal{E}_{\mathrm{H}}^{\varepsilon}[u]-e_{\mathrm{H}}^{\varepsilon}\right) d \mu_{N}+e_{\mathrm{H}}^{\varepsilon} \mu_{N}\left(S P_{-} \mathfrak{H}\right)-\frac{C L^{1+d / s+d / 2}}{N}
\end{aligned}
$$

where we have used the estimate $N_{L} \leq C L^{d / s+d / 2}$ of Lemma 3.3. Applying the variational principle $\mathcal{E}_{\mathrm{H}}^{\varepsilon}[u] \geq e_{\mathrm{H}}^{\varepsilon}$ and computing $\int d \mu_{N}$ explicitly using (3.10), we get

$$
\frac{1}{2} \operatorname{Tr}\left[P_{-}^{\otimes 2} H_{2}^{\varepsilon} P_{-}^{\otimes 2} \gamma_{N}^{(2)}\right] \geq e_{\mathrm{H}}^{\varepsilon} \sum_{k=2}^{N} \frac{k(k-1)}{N(N-1)} \operatorname{Tr}_{\mathfrak{H}^{k}}\left[G_{N, k}^{-}\right]-\frac{C L^{1+d / s+d / 2}}{N} .
$$

If $e_{\mathrm{H}}^{\varepsilon} \leq 0$ then we simply write

$$
\sum_{k=2}^{N} \frac{k(k-1)}{N(N-1)} \operatorname{Tr}_{\mathfrak{H}^{k}}\left[G_{N, k}^{-}\right] \leq \sum_{k=2}^{N} \operatorname{Tr}_{\mathfrak{H}^{k}}\left[G_{N, k}^{-}\right] \leq 1
$$

and we are done. If $e_{\mathrm{H}}^{\varepsilon}>0$, then we need to prove that $\int d \mu_{N} \simeq 1$ and for this we use the positive term

$$
\operatorname{Tr}\left[\left(P_{+} \otimes \mathbb{1}+\mathbb{1} \otimes P_{+}\right) \gamma_{N}^{(2)}\right]=2 \operatorname{Tr}\left[P_{+} \gamma_{N}^{(1)}\right]=2\left(1-\operatorname{Tr}\left[P_{-} \gamma_{N}^{(1)}\right]\right) .
$$


First, recall that by (3.8)

$$
\operatorname{Tr}\left[P_{-} \gamma_{N}^{(1)}\right]=\sum_{k=1}^{N} \frac{k}{N} \operatorname{Tr}_{\mathfrak{H}^{k}}\left[G_{N, k}^{-}\right] .
$$

Then, using Jensen's inequality, we have

$$
\begin{aligned}
\sum_{k=2}^{N} \frac{k(k-1)}{N(N-1)} \operatorname{Tr}_{\mathfrak{H}^{k}}\left[G_{N, k}^{-}\right] & =\frac{N}{N-1} \sum_{k=0}^{N} \frac{k^{2}}{N^{2}} \operatorname{Tr}_{\mathfrak{H}^{k}}\left[G_{N, k}^{-}\right]-\frac{\operatorname{Tr} P_{-} \gamma_{N}^{(1)}}{N-1} \\
& \geq \frac{N}{N-1}\left(\sum_{k=0}^{N} \frac{k}{N} \operatorname{Tr}_{\mathfrak{H}^{k}}\left[G_{N, k}^{-}\right]\right)^{2}-\frac{\operatorname{Tr} P_{-} \gamma_{N}^{(1)}}{N-1} \\
& =\frac{N}{N-1}\left(\operatorname{Tr} P_{-} \gamma_{N}^{(1)}\right)^{2}-\frac{\operatorname{Tr} P_{-} \gamma_{N}^{(1)}}{N-1}
\end{aligned}
$$

Therefore, denoting by

$$
\lambda:=\operatorname{Tr} P_{-} \gamma_{N}^{(1)} \leq 1
$$

the fraction of particles localized on the low energy states, we have the lower bound

$$
\frac{1}{2} \operatorname{Tr}\left[P_{-}^{\otimes 2} H_{2}^{\varepsilon} P_{-}^{\otimes 2} \gamma_{N}^{(2)}\right]+\frac{L}{4} \operatorname{Tr} P_{+} \gamma_{N}^{(1)} \geq \frac{N \lambda^{2}-\lambda}{N-1} e_{\mathrm{H}}^{\varepsilon}+\frac{L}{4}(1-\lambda)-\frac{C L^{1+d / s+d / 2}}{N} .
$$

When $L>8 N e_{\mathrm{H}}^{\varepsilon} /(N-1)$, the minimum of the right side is attained at $\lambda=1$. In dimension $d \geq 2$, we have $e_{\mathrm{H}}^{\varepsilon} \leq C N^{d \beta}$ and $L \geq C N^{d \beta} / \varepsilon$, and hence the condition $L>8 N e_{\mathrm{H}}^{\varepsilon} /(N-1)$ is always fulfilled for $N$ large enough. Similarly, if $d=1$ and $\beta>0$, we use that $\left|e_{\mathrm{H}}^{\varepsilon}\right| \leq C$ and $L \geq C \varepsilon^{-2}$. In all cases we get

$$
\frac{1}{2} \operatorname{Tr}\left[P_{-}^{\otimes 2} H_{2}^{\varepsilon} P_{-}^{\otimes 2} \gamma_{N}^{(2)}\right]+\frac{L}{4} \operatorname{Tr}\left[P_{+} \gamma_{N}^{(1)}\right] \geq e_{\mathrm{H}}^{\varepsilon}-\frac{C L^{1+d / s+d / 2}}{N}
$$

which is the desired inequality.

3.4. Conclusion: proof of Theorem 2.1. The upper bound in (2.15) is trivial, taking a factorized trial state for the $N$-body energy. To show the lower bound, let us consider a ground state $\gamma_{N}=\left|\Psi_{N}\right\rangle\left\langle\Psi_{N}\right|$ of $H_{N}$. From Lemma 3.6 and Lemma 3.8, we have the lower bound

$$
\frac{E(N)}{N} \geq e_{\mathrm{H}}^{\varepsilon}-C \frac{L^{1+d / s+d / 2}}{N}+\frac{1}{4} \operatorname{Tr}\left[P_{+} H_{1} \gamma_{N}^{(1)}\right]
$$

which gives the result for $L=C N^{d \beta} \varepsilon^{-1}$ in dimensions $d \geq 2$ and, if $\beta=0$, in dimension $d=1$. The last term in (3.23) is positive and will be useful later. For now we can just drop it from the lower bound.

If $d=1$ and $\beta>0$, we take $L=C \varepsilon^{-2}$. By Lemma 3.7 we have $\left|e_{\mathrm{H}}\right| \leq C$ and

$$
H_{2}^{\varepsilon}=(1-\varepsilon) H_{2}+\varepsilon\left(H_{1} \otimes \mathbb{1}+\mathbb{1} \otimes H_{1}-\left(w_{N}\right)_{-}\right) \geq(1-\varepsilon) H_{2}-\varepsilon C,
$$

which implies $e_{\mathrm{H}}^{\varepsilon} \geq e_{\mathrm{H}}-C \varepsilon$. Thus we find

$$
\frac{E(N)}{N} \geq e_{\mathrm{H}}-C \varepsilon-C \frac{\varepsilon^{-3-2 / s}}{N}+\frac{1}{4} \operatorname{Tr}\left[P_{+} H_{1} \gamma_{N}^{(1)}\right] .
$$

The estimate (2.13) then follows after optimizing with respect to $\varepsilon$. 


\section{The NLS LIMIT}

Now we explain how to go from the Hartree model to the NLS model and prove Proposition 2.3 and Theorems 2.4 and 2.5 .

4.1. From Hartree to NLS: proof of Proposition 2.3. The key observations are summarized in the following lemma.

Lemma 4.1 (Stability of the effective one-body functionals).

Let $d \leq 3$. We assume that (2.1), (2.2) and (2.3) hold true, and that $|x| w(x) \in L^{1}\left(\mathbb{R}^{d}\right)$. When $d=2,3$ we also assume that $w$ is stable (in the sense of (2.23) when $d=2$ and (2.20) when $d=3$ ).

Then the set of minimizers for $\mathcal{E}_{\mathrm{nls}}$ is non-empty and compact in the quadratic form domain of $H_{1}$. Also, for any normalized function $u \in L^{2}\left(\mathbb{R}^{d}\right)$ we have

$$
\int_{\mathbb{R}^{d}}|\nabla| u|(x)|^{2} d x \leq C\left(\mathcal{E}_{\mathrm{H}}[u]+C\right)
$$

and

$$
\left|\mathcal{E}_{\mathrm{H}}[u]-\mathcal{E}_{\mathrm{nls}}[u]\right| \leq C N^{-\beta}\left(1+\int_{\mathbb{R}^{d}}|\nabla| u|(x)|^{2} d x\right)^{2} .
$$

Proof. We start by proving (4.1). When $d=3$, since $w$ satisfies (2.20) the nonlinear term is positive, and therefore the inequality (4.1) follows immediately from the diamagnetic inequality $|(\nabla+i A) u| \geq|\nabla| u||$ and the assumption that $V \geq-C$.

If $d=2$, we use the stability assumption on $w$ to obtain, for $\eta$ small enough,

$$
\begin{aligned}
C+\mathcal{E}_{\mathrm{H}}(u) \geq & \int_{\mathbb{R}^{2}}|\nabla| u||^{2}+\frac{1}{2} \iint_{\mathbb{R}^{2} \times \mathbb{R}^{2}}|u(x)|^{2}|u(y)|^{2} w(x-y) d x d y \\
\geq & \frac{1}{1-\eta}\left(\int_{\mathbb{R}^{2}}|\nabla| u||^{2}+\frac{1-\eta}{2} \iint_{\mathbb{R}^{2} \times \mathbb{R}^{2}}|u(x)|^{2}|u(y)|^{2} w(x-y) d x d y\right) \\
& \quad+\left.\frac{\eta}{1-\eta} \int_{\mathbb{R}^{2}}|\nabla| u\right|^{2} \\
\geq & \frac{\eta}{1-\eta} \int_{\mathbb{R}^{2}}|\nabla| u||^{2} .
\end{aligned}
$$

Finally, in dimension $d=1$, we use Lemma 3.7 and obtain

$$
\mathcal{E}_{\mathrm{H}}[u]=\frac{1}{2}\left\langle u^{\otimes 2}, H_{2} u^{\otimes 2}\right\rangle \geq-C-C\left(\int_{\mathbb{R}} w^{-}\right)^{2}+\frac{1}{2}\left\langle u, H_{1} u\right\rangle \geq \frac{1}{2} \int_{\mathbb{R}}\left|u^{\prime}\right|^{2}-C .
$$

Next, we prove (4.2). We change variables to write

$$
\begin{aligned}
\mathcal{E}_{\mathrm{H}}[u]-\mathcal{E}_{\mathrm{nls}}[u] & =\frac{1}{2} \iint_{\mathbb{R}^{d} \times \mathbb{R}^{d}}|u(x)|^{2} w_{N}(x-y)|u(y)|^{2} d x d y-\frac{1}{2}\left(\int w\right) \int_{\mathbb{R}^{d}}|u|^{4} \\
& =\frac{1}{2} \iint_{\mathbb{R}^{d} \times \mathbb{R}^{d}}|u(x)|^{2} w(z)\left(\left|u\left(x+z N^{-\beta}\right)\right|^{2}-|u(x)|^{2}\right) d x d z \\
& =\frac{1}{2} \iint_{\mathbb{R}^{d} \times \mathbb{R}^{d}}|u(x)|^{2} w(z)\left(\int_{0}^{1} \nabla|u|^{2}\left(x+t z N^{-\beta}\right) \cdot z N^{-\beta} d t\right) d x d z
\end{aligned}
$$


and hence

$$
\left|\mathcal{E}_{\mathrm{H}}[u]-\mathcal{E}_{\mathrm{nls}}[u]\right| \leq N^{-\beta}\left(\int_{\mathbb{R}^{d}}|z||w(z)| d z\right)\left\||u|^{2} * \nabla|u|^{2}\right\|_{L^{\infty}\left(\mathbb{R}^{d}\right)} .
$$

In dimensions $d \leq 3$, we can write by the Young and Sobolev inequalities

$$
\left\|\left.|u|^{2} *|\nabla| u\right|^{2}\left|\left\|_{L^{\infty}\left(\mathbb{R}^{d}\right)} \leq\right\| u\left\|_{L^{6}}^{3}\right\| \nabla\right| u\left|\left\|_{L^{2}\left(\mathbb{R}^{d}\right)} \leq C\right\|\right| u \mid\right\|_{H^{1}\left(\mathbb{R}^{d}\right)}^{4},
$$

which concludes the proof of (4.2).

With Lemma 4.1 at hand, it is now easy to prove Proposition 2.3 .

Proof of Proposition 2.3. We first prove

$$
\left|e_{\mathrm{H}}-e_{\mathrm{nls}}\right| \leq C N^{-\beta} \text {. }
$$

Let $v$ be a minimizer for $e_{\text {nls }}$. Then $|v| \in H^{1}\left(\mathbb{R}^{d}\right)$ by (4.1). Consequently,

$$
e_{\mathrm{H}} \leq \mathcal{E}_{\mathrm{H}}(v) \leq \mathcal{E}_{\mathrm{nls}}(v)+C N^{-\beta}\|\mid v\|_{H^{1}\left(\mathbb{R}^{d}\right)}^{4}=e_{\mathrm{nls}}+C N^{-\beta} .
$$

Similarly, pick for every $N$ a minimizer $u_{N}$ for $e_{\mathrm{H}}$. By (4.1), $\left|u_{N}\right|$ is uniformly bounded in $H^{1}\left(\mathbb{R}^{d}\right)$. Hence

$$
e_{\mathrm{nls}} \leq \mathcal{E}_{\mathrm{nls}}\left(u_{N}\right) \leq \mathcal{E}_{\mathrm{H}}\left(u_{N}\right)+C N^{-\beta}\left\|\left|u_{N}\right|\right\|_{H^{1}\left(\mathbb{R}^{d}\right)}^{4} \leq e_{\mathrm{H}}+C N^{-\beta},
$$

which concludes the proof of (4.3).

Next we prove that $e_{\mathrm{H}} \rightarrow-\infty$ as $N \rightarrow \infty$ when $w$ is not stable in dimensions $d \geq 2$. Let $u \in C_{c}^{\infty}\left(\mathbb{R}^{d}\right)$ with support in the unit ball and $\|u\|_{L^{2}}=1$. Using as trial state $v_{N}(\bar{x})=$ $N^{d \beta / 2} u\left(N^{\beta} x\right)$, our assumption (2.2) on $V$ and the pointwise estimate

$$
\left|\nabla v_{N}+i A v_{N}\right|^{2} \leq(1+\varepsilon)\left|\nabla v_{N}\right|^{2}+\left(1+\varepsilon^{-1}\right)|A|^{2}\left|v_{N}\right|^{2},
$$

we find that

$$
\begin{aligned}
e_{\mathrm{H}} \leq C+(1+\varepsilon) & N^{2 \beta} \int_{\mathbb{R}^{d}}|\nabla u|^{2}+\left(1+\varepsilon^{-1}\right) N^{d \beta}\|u\|_{L^{\infty}} \int_{|x| N^{\beta} \leq 1}|A|^{2}+C N^{-S \beta} \int_{\mathbb{R}^{d}}|x|^{S}|u|^{2} \\
& +\frac{N^{d \beta}}{2} \iint_{\mathbb{R}^{d} \times \mathbb{R}^{d}}|u(x)|^{2} w(x-y)|u(y)|^{2} d x d y \\
& =(1+\varepsilon) N^{2 \beta} \int_{\mathbb{R}^{d}}|\nabla u|^{2}+\frac{N^{d \beta}}{2} \iint_{\mathbb{R}^{d} \times \mathbb{R}^{d}}|u(x)|^{2} w(x-y)|u(y)|^{2} d x d y+o\left(N^{d \beta}\right) .
\end{aligned}
$$

If $d \geq 3$ and $w$ is not classically stable, we choose $u$ to have

$$
\iint_{\mathbb{R}^{d} \times \mathbb{R}^{d}}|u(x)|^{2} w(x-y)|u(y)|^{2} d x d y<0,
$$

and we conclude that $\lim _{N \rightarrow \infty} e_{\mathrm{H}}=\lim _{N \rightarrow \infty} E(N) / N=-\infty$ since $E(N) / N \leq e_{\mathrm{H}}$. If $d=2$, we take $\varepsilon^{2}=\int_{|x| \leq N^{-\beta}}|A|^{2}$ and obtain

$$
\limsup _{N \rightarrow \infty} \frac{e_{\mathrm{H}}}{N^{2 \beta}} \leq \inf _{\substack{u \in H^{1}\left(\mathbb{R}^{2}\right) \\\|u\|_{L^{2}\left(\mathbb{R}^{2}\right)=1}}}\left(\int_{\mathbb{R}^{2}}|\nabla u|^{2}+\frac{1}{2} \iint_{\mathbb{R}^{2} \times \mathbb{R}^{2}}|u(x)|^{2} w(x-y)|u(y)|^{2} d x d y\right) .
$$

The right side is strictly negative by assumption. 
4.2. Convergence of the many-body energy: proof of Theorem 2.4. In the cases $d=1, d=2$ with $w$ stable, the result immediately follows from Theorem 2.1, Remark 2.2 and Proposition 2.3. If $w-\eta|w|$ is stable for some $0<\eta<1$, so is $w-\varepsilon|w|$ for all $0 \leq \varepsilon \leq \eta$. Then we can apply Proposition 2.3 with $w$ replaced by $w-\varepsilon|w|$ for some $\varepsilon<1$ to be tuned later on and get

$$
\left|e_{\mathrm{nls}}^{\varepsilon}-e_{\mathrm{H}}^{\varepsilon}\right| \leq C N^{-\beta}
$$

where $e_{\mathrm{nls}}^{\varepsilon}$ is the NLS minimization problem, with $a=\int w$ replaced by $\int w-\varepsilon \int|w|$. Arguing as in the proof of Lemma 4.1, it is not difficult to see that $\left|e_{\mathrm{nls}}^{\varepsilon}-e_{\mathrm{nls}}\right| \leq C \varepsilon$. Therefore Theorem 2.1 provides the bound

$$
e_{\mathrm{nls}}+C N^{-\beta} \geq \frac{E(N)}{N} \geq e_{\mathrm{nls}}-C \varepsilon-C N^{-\beta}-C \frac{\varepsilon^{-1-d / 2-d / s}}{N^{1-d \beta(1+d / 2+d / s)}} .
$$

Optimizing over $\varepsilon$ gives (2.29).

4.3. Convergence of states: proof of Theorem 2.5. We split the proof in four steps for clarity.

Step 1, strong compactness of density matrices. We first note that $\gamma_{N}^{(n)}$ is by definition bounded in the trace-class, so that we can extract a subsequence along which

$$
\gamma_{N}^{(n)} \rightarrow_{*} \gamma^{(n)}
$$

as $N \rightarrow \infty$. Modulo a diagonal extraction argument, one can assume that the convergence is along the same subsequence for any $n$. We now argue that the convergence is actually strong. We start by proving that

$$
\operatorname{Tr}\left[H_{1} \gamma_{N}^{(1)}\right]=\operatorname{Tr}\left[\left(-(\nabla+i A)^{2}+V\right) \gamma_{N}^{(1)}\right] \leq C,
$$

independently of $N$. To this end, pick some $\alpha>0$ and define

$$
H_{N, \alpha}=\sum_{j=1}^{N}\left(-\left(\nabla+i A\left(x_{j}\right)\right)^{2}+V\left(x_{j}\right)\right)+\frac{1+\alpha}{N-1} \sum_{1 \leq i<j \leq N} N^{d \beta} w\left(N^{\beta}\left(x_{i}-x_{j}\right)\right) .
$$

Noticing for instance that

$$
(1+\eta / 4)(w-\eta / 4|w|) \geq w-\left(\eta / 2+\eta^{2} / 16\right)|w| \geq w-\eta|w|,
$$

we can apply the results of Theorem 2.4 with $H_{N}$ replaced by $H_{N, \alpha}$. We find in particular that $H_{N, \alpha} \geq-C N$ and deduce that

$$
e_{\mathrm{nls}}+o(1) \geq \frac{\left\langle\Psi_{N}, H_{N} \Psi_{N}\right\rangle}{N} \geq-C(1+\alpha)^{-1}+\frac{\alpha}{1+\alpha} \operatorname{Tr}\left[H_{1} \gamma_{N}^{(1)}\right]
$$

Hence (4.6) holds true. Since $H_{1}=-(\nabla+i A)^{2}+V$ has a compact resolvent, (4.5) and (4.6) imply that, up to a subsequence, $\gamma_{N}^{(1)}$ converges strongly in the trace class. By [32, Corollary 2.4], $\gamma_{N}^{(n)}$ converges strongly as well for all $n \geq 1$.

Step 2, introducing the limit measure. Now we extract some useful information from the proof of Theorem 2.1. We shall use the same notation as in Section 3 (with the same choices for $L$ and $\varepsilon$ that were made later in the proof). For simplicity we denote by

$$
r_{N}:=N^{-\beta}+N^{-\frac{1}{4+2 / s}} \mathbb{1}(d=1)+N^{-\frac{1-d \beta(1+d / 2+d / s)}{2+d / s+d / 2}} \mathbb{1}(d=2,3)
$$


the best error bound that we have derived on $\left|E(N) / N-e_{\mathrm{nls}}\right|$.

Let $d \mu_{N}$ be defined as in Lemma 3.4, which is such that

$$
\mu_{N}\left(S P_{-} \mathfrak{H}\right)=\operatorname{Tr}\left[P_{-}^{\otimes 2} \gamma_{N}^{(2)} P_{-}^{\otimes 2}\right]
$$

Our arguments of Section 3 actually imply that $\mu_{N}\left(S P_{-} \mathfrak{H}\right) \rightarrow 1$ but we will recover this fact here. We have

$$
\left.\operatorname{Tr}\left|P_{-}^{\otimes 2} \gamma_{N}^{(2)} P_{-}^{\otimes 2}-\int_{S P_{-} \mathfrak{H}}\right| u^{\otimes 2}\right\rangle\left\langle u^{\otimes 2}\left|d \mu_{N}(u)\right| \leq \frac{8 N_{L}}{N} \leq C \frac{L^{1+d / s+d / 2}}{N} \rightarrow 0 .\right.
$$

On the other hand, the estimate (3.22) and the error bounds provide a control on the number of excited particles:

$$
1-\mu_{N}\left(S P_{-} \mathfrak{H}\right)=\operatorname{Tr}\left[\left(1-P_{-}^{\otimes 2}\right) \gamma_{N}^{(2)}\right] \leq 2 \operatorname{Tr}\left[P_{+} \gamma_{N}^{(1)}\right] \leq \frac{r_{N}}{L} .
$$

Therefore, by the triangle and Cauchy-Schwarz inequalities, we find

$$
\left.\operatorname{Tr}\left|\gamma_{N}^{(2)}-\int_{S P_{-} \mathfrak{H}}\right| u^{\otimes 2}\right\rangle\left\langle u^{\otimes 2}\left|d \mu_{N}(u)\right| \leq C \frac{L^{1+d / s+d / 2}}{N}+C \sqrt{\frac{r_{N}}{L}} .\right.
$$

Next, we denote $P_{K}$ the spectral projector of $H_{1}$ onto energies below a cut-off $K$. Since $\gamma_{N}^{(2)} \rightarrow \gamma^{(2)}$ and $P_{K} \rightarrow \mathbb{1}$ we deduce from the above

$$
\lim _{K \rightarrow \infty} \lim _{N \rightarrow \infty} \mu_{N}\left(S P_{K} \mathfrak{H}\right)=1 .
$$

This tightness condition allows us to use Prokhorov's theorem and [56, Lemma 1] to ensure that, up to extraction of a subsequence, $\mu_{N}$ converges weakly to a measure $\mu$ in the ball $B \mathfrak{H}$. After passing to the weak limit, we find that

$$
\gamma^{(2)}=\int_{B \mathfrak{H}}\left|u^{\otimes 2}\right\rangle\left\langle u^{\otimes 2}\right| d \mu(u) .
$$

Since $\mu(B \mathfrak{H}) \leq 1$ and $\operatorname{Tr} \gamma^{(2)}=1$ by the strong convergence of $\gamma_{N}^{(2)}$, we conclude that $\mu$ is supported on the sphere $S \mathfrak{H}$.

Step 3, the limit measure charges only NLS minimizers. Going back to our proof in the previous sections and using that

we deduce the bound

$$
\mu_{N}\left(S P_{-} \mathfrak{H}\right)=1+O\left(\frac{r_{N}}{L}\right)
$$

$$
\int_{S P_{-} \mathfrak{H}}\left(\mathcal{E}_{\mathrm{H}}^{\varepsilon}[u]-e_{\mathrm{H}}^{\varepsilon}\right) d \mu_{N}(u) \leq C r_{N}
$$

on the term that we had neglected in (3.20). By Lemma 4.1, this implies that, for $B$ a large enough (but fixed) constant,

and

$$
\frac{B^{2}}{C} \int_{\|\nabla|u|\|_{H^{1}} \geq B} d \mu_{N}(u) \leq \int_{\|\nabla|u|\|_{H^{1}} \geq B}\left(\mathcal{E}_{\mathrm{H}}^{\varepsilon}[u]-e_{\mathrm{H}}^{\varepsilon}\right) d \mu_{N}(u) \leq C r_{N} .
$$

$\int_{\|\nabla|u|\|_{L^{2}} \leq B}\left(\mathcal{E}_{\mathrm{nls}}[u]-e_{\mathrm{nls}}\right) d \mu_{N} \leq C\left(1+B^{4}\right)\left(\varepsilon+N^{-\beta}\right)+\int_{\|\nabla|u|\|_{L^{2}} \leq B}\left(\mathcal{E}_{\mathrm{H}}[u]-e_{\mathrm{H}}\right) d \mu_{N}(u) \leq C r_{N}$.

Passing to the limit $N \rightarrow \infty$, it is now clear that $\mu$ has its support on $\mathcal{M}_{\text {nls }}$. 
At this stage, from (4.8) and the convergence of $\mu_{N}$ we have, along a subsequence

$$
\gamma_{N}^{(2)} \rightarrow \int_{\mathcal{M}_{\text {nls }}}\left|u^{\otimes 2}\right\rangle\left\langle u^{\otimes 2}\right| d \mu(u),
$$

strongly in the trace-class, where $\mu$ is a probability measure supported on $\mathcal{M}_{\text {nls }}$. Taking a partial trace we also have

$$
\gamma_{N}^{(1)} \rightarrow \int_{\mathcal{M}_{\mathrm{nls}}}|u\rangle\langle u| d \mu(u)
$$

Step 4, higher order density matrices. There remains to prove that, for any $n>2$,

$$
\gamma_{N}^{(n)} \rightarrow \int_{\mathcal{M}_{\mathrm{nls}}}\left|u^{\otimes n}\right\rangle\left\langle u^{\otimes n}\right| d \mu(u)
$$

strongly in the trace-class when $N \rightarrow \infty$. In view of the definition of $\mu$ this follows from the estimate

$$
\left.\operatorname{Tr}\left|\gamma_{N}^{(n)}-\int_{S P_{-} \mathfrak{H}}\right| u^{\otimes n}\right\rangle\left\langle u^{\otimes n}\left|d \mu_{N}(u)\right| \rightarrow 0 .\right.
$$

To see that (4.9) holds we first define a measure approximating $\gamma_{N}^{(n)}$, as indicated in Remark 3.5. Arguing as in the proof of Lemma 3.4 we have

$$
\left.\operatorname{Tr}_{\mathfrak{H}^{n}}\left|P_{-}^{\otimes n} \gamma_{N}^{(n)} P_{-}^{\otimes n}-\int_{S P_{-} \mathfrak{H}}\right| u^{\otimes n}\right\rangle\left\langle u^{\otimes n}\left|d \mu_{N}^{n}(u)\right| \leq C \frac{n N_{L}}{N}\right.
$$

where

$$
d \mu_{N}^{n}(u)=\sum_{k=n}^{N}\left(\begin{array}{l}
N \\
n
\end{array}\right)^{-1}\left(\begin{array}{l}
k \\
n
\end{array}\right) d \mu_{N, k}(u)
$$

and $d \mu_{N, k}$ is the same measure as in (3.10). An estimate similar to (4.7) next shows that

$$
\left.\operatorname{Tr}_{\mathfrak{H}^{n}}\left|\gamma_{N}^{(n)}-\int_{S P_{-} \mathfrak{H}}\right| u^{\otimes n}\right\rangle\left\langle u^{\otimes n}\left|d \mu_{N}^{n}(u)\right| \rightarrow 0 .\right.
$$

Using the easy bound (see [32, Section 2])

$$
\left(\begin{array}{l}
N \\
n
\end{array}\right)^{-1}\left(\begin{array}{l}
k \\
n
\end{array}\right)=\left(\frac{k}{N}\right)^{n}+O\left(N^{-1}\right)
$$

together with the triangle inequality and Schur's formula (see Remark 3.2) we next deduce from (4.10) that

$$
\begin{aligned}
\left.\operatorname{Tr}\left|\gamma_{N}^{(n)}-\int_{S P_{-} \mathfrak{H}}\right| u^{\otimes n}\right\rangle\left\langle u^{\otimes n}\left|d \mu_{N}(u)\right|\right. & \leq \sum_{k=0}^{N}\left(\left(\frac{k}{N}\right)^{2}-\left(\frac{k}{N}\right)^{n}\right) \operatorname{Tr}_{\mathfrak{H}^{k}}\left[G_{N, k}^{-}\right] \\
& +\sum_{k=0}^{n-1}\left(\frac{k}{N}\right)^{n} \operatorname{Tr}_{\mathfrak{H}^{k}}\left[G_{N, k}^{-}\right] \\
& +\sum_{k=0}^{2}\left(\frac{k}{N}\right)^{2} \operatorname{Tr}_{\mathfrak{H}^{k}}\left[G_{N, k}^{-}\right]+o(1) .
\end{aligned}
$$


Finally, combining our previous estimates gives

$$
\sum_{k=2}^{N}\left(\frac{k}{N}\right)^{2} \operatorname{Tr}_{\mathfrak{H}^{k}}\left[G_{N, k}^{-}\right] \rightarrow 1
$$

and using (3.9) we in fact have

$$
\sum_{k=0}^{N}\left(\frac{k}{N}\right)^{2} \operatorname{Tr}_{\mathfrak{H}^{k}}\left[G_{N, k}^{-}\right] \rightarrow 1
$$

Then by Jensen's inequality

$$
1 \geq \sum_{k=0}^{N}\left(\frac{k}{N}\right)^{n} \operatorname{Tr}_{\mathfrak{H}^{k}}\left[G_{N, k}^{-}\right] \geq\left(\sum_{k=0}^{N}\left(\frac{k}{N}\right)^{2} \operatorname{Tr}_{\mathfrak{H}^{k}}\left[G_{N, k}^{-}\right]\right)^{n / 2} \rightarrow 1 .
$$

Inserting this and (3.9) in (4.12) concludes the proof of (4.9) and thus that of the theorem.

We finally note that our method can give quantitative estimates on reduced density matrices in some special cases:

Remark 4.2 (The case of a stable NLS functional). If a stability estimate of the form

$$
\mathcal{E}_{\mathrm{nls}}[u] \geq e_{\mathrm{nls}}+c \inf _{v \in \mathcal{M}_{\mathrm{nls}}}\|u-v\|^{2}
$$

holds at the level of the NLS functional, for some norm $\|\cdot\|$ (say the $L^{2}$ norm), it is easy to see that the previous method leads to quantitative estimates on density matrices. In particular, if the NLS minimizer is unique (up to a constant phase), non-degenerate, and (4.13) holds, one may obtain quantitative bounds on the depletion of the condensate. Indeed, it immediately follows from the above considerations and (4.13) that

$$
\int_{u \in S P_{-} \mathfrak{H}} \||u\rangle\left\langle u|-| u_{\mathrm{nls}}\right\rangle\left\langle u_{\mathrm{nls}}\right| \|_{\mathfrak{S}^{2}}^{2} d \mu_{N}(u) \leq r_{N}
$$

where $\mathfrak{S}^{2}$ denotes the Hilbert-Schmidt class. Then, using Jensen's inequality together with the fact that $\left|u_{\mathrm{nls}}\right\rangle\left\langle u_{\mathrm{nls}}\right|$ is a rank-one projection we have

$$
\| \int_{u \in S P_{-} \mathfrak{H}}|u\rangle\left\langle u\left|d \mu_{N}(u)-\right| u_{\mathrm{nls}}\right\rangle\left\langle u_{\mathrm{nl}}\right| \|_{\mathfrak{S}^{1}} \leq C \sqrt{r_{N}}
$$

and thus, in view of our previous bounds,

$$
\| \gamma_{N}^{(n)}-\left|u_{\mathrm{nls}}^{\otimes n}\right\rangle\left\langle u_{\mathrm{nls}}^{\otimes n}\right| \|_{\mathfrak{S}^{1}\left(L^{2}\left(\mathbb{R}^{3}\right)\right)} \leq C_{n} \sqrt{r_{N}}
$$

where $C_{n}$ depends only on $n$.

Since we do not want to rely on assumptions such as (4.13) we do not pursue in this direction. One may consult e.g. 9] or [24, Section 6] for discussions of estimates of the form (4.13). $\triangle$ 


\section{REFERENCES}

[1] R. Adami, C. Bardos, F. Golse, and A. Teta, Towards a rigorous derivation of the cubic NLSE in dimension one, Asympt. Anal., 40 (2004), pp. 93-108.

[2] A. Aftalion, Vortices in Bose-Einstein Condensates, vol. 67 of Progress in nonlinear differential equations and their applications, Springer, 2006.

[3] A. Aftalion, R. Jerrard, and J. Royo-Letelier, Non existence of vortices in the small density region of a condensate, J. Funct. Anal., 260 (2011), pp. 2387-2406.

[4] Z. Ammari And F. NiER, Mean field limit for bosons and infinite dimensional phase-space analysis, Annales Henri Poincaré, 9 (2008), pp. 1503-1574. 10.1007/s00023-008-0393-5.

[5] W. Aschbacher, J. Fröhlich, G. Graf, K. Schnee, and M. Troyer, Symmetry breaking regime in the nonlinear hartree equation, J. Math. Phys., 43 (2002), pp. 3879-3891.

[6] C. Bardos, F. Golse, And N. J. Mauser, Weak coupling limit of the N-particle Schrödinger equation, Methods Appl. Anal., 7 (2000), pp. 275-293.

[7] R. Benguria And E. H. Lieb, Proof of the Stability of Highly Negative Ions in the Absence of the Pauli Principle, Physical Review Letters, 50 (1983), pp. 1771-1774.

[8] J.-B. Bru, M. Correggi, P. Pickl, and J. Yngvason, The TF limit for rapidly rotating bose gases in anharmonic traps, Comm. Math. Phys., 280 (2008), pp. 517-544.

[9] E. Carlen, R. Frank, And E. Lieb, Stability estimates for the lowest eigenvalue of a Schrödinger operator, Geom. Func. Anal., (2014).

[10] X. Chen And J. Holmer, Focusing quantum many-body dynamics: The rigorous derivation of the 1d focusing cubic nonlinear Schrödinger equation, preprint arXiv 1308.3895, (2013).

[11] G. ChiRIBella, On quantum estimation, quantum cloning and finite quantum de Finetti theorems, in Theory of Quantum Computation, Communication, and Cryptography, vol. 6519 of Lecture Notes in Computer Science, Springer, 2011.

[12] M. Christandl, R. König, G. Mitchison, And R. Renner, One-and-a-half quantum de Finetti theorems, Comm. Math. Phys., 273 (2007), pp. 473-498.

[13] J. Combes, R. Schrader, and R. Seiler, Classical bounds and limits for energy distributions of Hamilton operators in electromagnetic fields, Annals of Physics, 111 (1978), pp. 1 - 18.

[14] N. Cooper, Rapidly rotating atomic gases, Adv. Phys., 57 (2008), pp. 593-616.

[15] M. Correggi, F. Pinsker, N. Rougerie, and J. Yngvason, Critical rotational speeds in the GrossPitaevskii theory on a disc with Dirichlet boundary conditions, J. Stat. Phys., 143 (2011), pp. 261-305.

[16] — Critical rotational speeds for superfluids in homogeneous traps, J. Math. Phys., 53 (2012), p. 095203.

[17] M. Correggi, T. Rindler-Daller, And J. Yngvason, Rapidly rotating Bose-Einstein condensates in strongly anharmonic traps, J. Math. Phys., 48 (2007), p. 042104.

[18] J. Dalibard, F. Gerbier, G. Juzeliūnas, and P. ÖHBerg, Artificial gauge potentials for neutral atoms, Rev. Mod. Phys., 83 (2011), p. 1523.

[19] L. ERdös, B. Schlein, And H.-T. YAU, Derivation of the cubic non-linear Schrödinger equation from quantum dynamics of many-body systems, Invent. Math., 167 (2007), pp. 515-614.

[20] L. Erdős, B. Schlein, And H.-T. YAu, Rigorous derivation of the Gross-Pitaevskii equation with a large interaction potential, J. Amer. Math. Soc., 22 (2009), pp. 1099-1156.

[21] M. Fannes, H. Spohn, And A. Verbeure, Equilibrium states for mean field models, J. Math. Phys., 21 (1980), pp. 355-358.

[22] M. Fannes And C. Vandenplas, Finite size mean-field models, J. Phys. A, 39 (2006), pp. 13843-13860.

[23] A. Fetter, Rotating trapped Bose-Einstein condensates, Rev. Mod. Phys., 81 (2009), p. 647.

[24] R. L. Frank, Ground states of semi-linear PDEs. Lecture notes from the "Summer school on Current Topics in Mathematical Physics", Marseille 2014.

[25] J. Fröhlich, A. Knowles, And S. Schwarz, On the mean-field limit of bosons with Coulomb two-body interaction, Commun. Math. Phys., 288 (2009), pp. 1023-1059.

[26] P. Grech And R. Seiringer, The excitation spectrum for weakly interacting bosons in a trap, Comm. Math. Phys., 322 (2013), pp. 559-591.

[27] Y. Guo And R. Seiringer, On the mass concentration for Bose-Einstein condensates with attractive interactions, Lett. Math. Phys., 104 (2014), pp. 141-156. 
[28] A. Harrow, The church of the symmetric subspace, preprint arXiv 1308.6595, (2013).

[29] R. L. Hudson And G. R. Moody, Locally normal symmetric states and an analogue of de Finetti's theorem, Z. Wahrscheinlichkeitstheorie und Verw. Gebiete, 33 (1975/76), pp. 343-351.

[30] M. Lewin, Geometric methods for nonlinear many-body quantum systems, J. Funct. Anal., 260 (2011), pp. 3535-3595.

[31] M. Lewin, P. T. NAm, And N. Rougerie, Remarks on the quantum de Finetti theorem for bosonic systems, Appl. Math. Res. Express (AMRX), (2015), pp. 48-63

[32] - Derivation of Hartree's theory for generic mean-field Bose systems, Advances in Mathematics, 254 (2014).

[33] M. Lewin, P. T. Nam, S. Serfaty, and J. P. Solovej, Bogoliubov spectrum of interacting Bose gases, Comm. Pure Appl. Math., 68 (2015), pp. 413-471.

[34] E. H. Lieb AND W. Liniger, Exact analysis of an interacting Bose gas. I. The general solution and the ground state, Phys. Rev. (2), 130 (1963), pp. 1605-1616.

[35] E. H. Lieb And R. Seiringer, Derivation of the Gross-Pitaevskii equation for rotating Bose gases, Commun. Math. Phys., 264 (2006), pp. 505-537.

[36] — The Stability of Matter in Quantum Mechanics, Cambridge Univ. Press, 2010.

[37] E. H. Lieb, R. Seiringer, J. P. Solovej, And J. Yngvason, The mathematics of the Bose gas and its condensation, Oberwolfach Seminars, Birkhäuser, 2005.

[38] E. H. Lieb, R. Seiringer, And J. Yngvason, Bosons in a trap: A rigorous derivation of the GrossPitaevskii energy functional, Phys. Rev. A, 61 (2000), p. 043602.

[39] - A rigorous derivation of the Gross-Pitaevskii energy functional for a two-dimensional Bose gas, Commun. Math. Phys., 224 (2001), pp. 17-31.

[40] E. H. Lieb, R. Seiringer, And J. Yngvason, Justification of c-Number Substitutions in Bosonic Hamiltonians, Phys. Rev. Lett., 94 (2005), p. 080401.

[41] E. H. LIEB AND H.-T. YAU, The Chandrasekhar theory of stellar collapse as the limit of quantum mechanics, Commun. Math. Phys., 112 (1987), pp. 147-174.

[42] E. H. Lieb And J. Yngvason, Ground state energy of the low density Bose gas, Phys. Rev. Lett., 80 (1998), pp. 2504-2507.

[43] M. MAEDA, On the symmetry of the ground states of nonlinear Schrödinger equation with potential, Adv. Nonlinear Stud., 10 (2010), pp. 895-925.

[44] C. Pethick And H. Smith, Bose-Einstein Condensation of Dilute Gases, Cambridge University Press, 2001.

[45] P. PickL, Derivation of the time dependent Gross Pitaevskii equation with external fields, Rev. Math. Phys., 27 (2015), pp. 1550003.

[46] - Derivation of the time dependent Gross-Pitaevskii equation without positivity condition on the interaction, J. Stat. Phys., 140 (2010), pp. 76-89.

[47] —, A simple derivation of mean-field limits for quantum systems, Lett. Math. Phys., 97 (2011), pp. $151-164$

[48] L. Pitaevskit and S. Stringari, Bose-Einstein Condensation, Oxford Science Publications, Oxford, 2003.

[49] G. A. RagGio And R. F. Werner, Quantum statistical mechanics of general mean field systems, Helv. Phys. Acta, 62 (1989), pp. 980-1003.

[50] I. Rodnianski And B. Schlein, Quantum fluctuations and rate of convergence towards mean field dynamics, Commun. Math. Phys., 291 (2009), pp. 31-61.

[51] D. Ruelle, Statistical mechanics. Rigorous results, Singapore: World Scientific. London: Imperial College Press , 1999.

[52] R. Seiringer, Gross-Pitaevskii theory of the rotating Bose gas, Commun. Math. Phys., 229 (2002), pp. 491-509.

[53] R. SeIRInger, The excitation spectrum for weakly interacting bosons, Commun. Math. Phys., 306 (2011), pp. 565-578.

[54] R. Seiringer, J. Yngvason, And V. A. Zagrebnov, Disordered Bose-Einstein condensates with interaction in one dimension, J. Stat. Mech., 2012 (2012), p. P11007.

[55] B. Simon, Functional integration and quantum physics, AMS Chelsea Publishing, Providence, RI, second ed., 2005. 
[56] A. Skorokhod, Integration in Hilbert space, Ergebnisse der Mathematik und ihrer Grenzgebiete, Springer-Verlag, 1974.

[57] E. Størmer, Symmetric states of infinite tensor products of $C^{*}$-algebras, J. Functional Analysis, 3 (1969), pp. 48-68.

[58] M. I. Weinstein, Nonlinear Schrödinger equations and sharp interpolation estimates, Comm. Math. Phys., 87 (1983), pp. 567-576.

[59] R. F. WERner, Large deviations and mean-field quantum systems, in Quantum probability \& related topics, QP-PQ, VII, World Sci. Publ., River Edge, NJ, 1992, pp. 349-381.

[60] J. Zhang, Stability of attractive Bose-Einstein condensates, J. Stat. Phys., 101 (2000), pp. 731-746.

CNRS \& Laboratoire de Mathématiques (UMR 8088), Université de Cergy-Pontoise, F-95000 Cergy-Pontoise, France.

E-mail address: mathieu.lewin@math.cnrs.fr

IST Austria, Am Campus 1, 3400 Klosterneuburg, Austria

E-mail address: pnam@ist.ac.at

CNRS \& Université Grenoble Alpes, LPMmC (UMR 5493), B.P. 166, F-38 042 Grenoble, FRANCE

E-mail address: nicolas.rougerie@grenoble.cnrs.fr 